\title{
Estrogen-related receptor alpha and Rplp1-dependent translation coordinately regulate starvation response and decrease NASH progression
}

Madhulika Tripathi ${ }^{1 \#}$, Karine Gauthier ${ }^{2 \#}$, Reddemma Sandireddy ${ }^{1}$, Jin Zhou ${ }^{1}$, Kabilesh Arul $^{1}$, Keziah Tikno ${ }^{1}$, Sung-Hee Park ${ }^{3}$, Yajun $\mathrm{Wu}^{4}$, Boon-Huat Bay ${ }^{4}$, Vincent Giguere $^{5}$, Pierce Kah Hoe Chow ${ }^{6}$, Donald P. McDonnell ${ }^{3}$, Paul M. Yen ${ }^{1^{*}}$, Brijesh K. Sing ${ }^{1^{*}}$

${ }^{1}$ Laboratory of Hormonal Regulation, Cardiovascular and Metabolic Disorders Program, Duke-National University of Singapore (NUS) Medical School, Singapore 169857, Singapore; ${ }^{2}$ Institut de Génomique Fonctionnelle de Lyon, Université de Lyon, Université Lyon 1, CNRS, Ecole Normale Supérieure de Lyon, 46 Allée d'Italie, 69364 Lyon Cedex 07, France; ${ }^{3}$ Department of Pharmacology and Cancer Biology, Duke University School of Medicine, C238A Levine Science Research Center, Durham, NC 27710, USA; ${ }^{4}$ Department of Anatomy, Yong Loo Lin School of Medicine, NUS, Singapore 117594. ${ }^{5}$ Goodman Cancer Research Centre, McGill University, 1160 Pine Avenue West, Montreal, Québec H3A 1A3, Canada. ${ }^{6}$ Dept of Surgery, Singapore General Hospital and Dept. of Surgical Oncology, National Cancer Centre, Singapore-169608.

\#These authors contributed equally

${ }^{\star}$ Corresponding authors: Dr. Brijesh Kumar Singh, Cardiovascular and Metabolic Disorders Program, Duke-National University of Singapore (NUS) Medical School, Singapore 169857, Singapore.singhbrijeshk@duke-nus.edu.sg 
Prof. Paul M. Yen, Cardiovascular and Metabolic Disorders Program, Duke-National

University of Singapore (NUS) Medical School, Singapore 169857, Singapore.

paul.yen@duke-nus.edu.sg

Keywords: Estrogen related receptor alpha; starvation, ribosome; translation;

lysosome; autophagy; non-alcoholic fatty liver disease (NAFLD), non-alcoholic

steatohepatitis (NASH)

Conflict of interest statement: Authors have no conflict of interests.

Funding details: This research was funded by the Ministry of Health (MOH), and National Medical Research Council (NMRC), Singapore, grant number NMRC/OFYIRG/0002/2016 and MOH-000319 (MOH-OFIRG19may-0002) to BKS; NMRC/OFYIRG/077/2018 to MT; and CSAI19may-0002 to PMY; Duke-NUS Medical School and Estate of Tan Sri Khoo Teck Puat Khoo Pilot Award (Collaborative) Duke-NUS-KP(Coll)/2018/0007A to JZ.

Author's contributions: MT, BKS, experimental design, execution and data analysis, and performed animal work; KG, performed animal experiments related to ESRRA knockout mice; KT, RS, KA, performed wet lab work; JZ, helped generating hepatic-ESRRA overexpressing mice; VG, generated ESRRA knockout mice and finalized manuscript; SP, DPM, shared C29, provided experimental suggestions and finalized manuscript; YW, BHB, performed EM and finalized manuscript; MT, BKS, PMY, drafted and finalized the manuscript, and provided financial support to this study.

Data availability: The data supporting the findings of this study are available within the article and/or supplementary materials. 


\section{Abstract}

Although general translation declines during fasting, maintaining the translation of a subset or proteins is necessary for metabolic homeostasis and cell viability. Using unbiased proteome analysis of hepatic cells during starvation, we identified a novel pathway in which Esrra-mediated transcription of Rplp1-dependent translation of lysosomal proteins declined during early starvation and recovered after prolonged starvation to restore autophagy-lysosome function. Interestingly, hepatic EsrraRplp1-dependent translation rate of lysosomal proteins also was impaired in patients and mice with non-alcoholic steatohepatitis (NASH), and translational response to starvation was dysregulated in mice with NASH. Remarkably, activation of Esrra pharmacologically, genetically, or by alternate day fasting restored protein translation, increased expression of lysosomal proteins, induced autophagy, and reduced lipotoxicity, inflammation, and fibrosis in cell culture and in vivo models of $\mathrm{NASH}$. Thus, hepatic Esrra is essential for ribosome-dependent translation of lysosomal proteins during starvation, and prevention of lipotoxicity and progression in $\mathrm{NASH}$. 


\section{Lay summary $\square$}

Fasting for weight loss improves non-alcoholic fatty liver disease (NAFLD) and non-alcoholic steatohepatitis (NASH); however, the mechanism is not well understood. Our study shows that a nuclear protein, estrogen related receptor alpha (Esrra), increases ribosome-mediated translation of autophagy and lysosome proteins during chronic starvation to maintain essential metabolic pathways for cell survival. Surprisingly, this translational pathway is impaired during NASH with reduced lysosome-autophagy activity accompanied by increased inflammation and fibrosis gene expression in the liver. $\square$

Pharmacologic, genetic and dietary activation of Esrra decreases lipidmediated toxicity in liver cells as well as inflammation and fibrosis in livers from mice with NASH. These findings suggest that the Esrra-ribosome-lysosome pathway is important for liver response to fasting and NASH and thus may be a good therapeutic target for the treatment of NASH. 


\section{Introduction}

Nonalcoholic fatty liver disease (NAFLD) is the leading chronic liver disease with a global prevalence of approximately $25 \%$ among adults worldwide ${ }^{1,2,3}$. It typically begins with hepatosteatosis, and can progress to nonalcoholic steatohepatitis (NASH), that in turn, can further advance to cirrhosis with increased risk for hepatocellular carcinoma ${ }^{3,4} . \square$ It is commonly associated with diabetes, dyslipidemia and obesity and is associated with increased risk for heart disease and renal failure. Although there are no pharmacologic therapies for NAFLD/NASH, fasting has been shown to be beneficial in reducing body fat during obesity, increasing insulin sensitivity in diabetes, and reducing hepatosteatosis, inflammation, and fibrosis in NAFLD ${ }^{5,6,7,8}$. However, acute fasting paradoxically increases hepatic steatosis $^{9,10,11}$. Thus, the role of fasting and its beneficial effect(s) on hepatosteatosis and NASH progression are not well understood at the molecular and cellular level.

Normal cellular homeostasis requires a proper balance between synthesis and degradation of proteins in response to changing environmental conditions.

Transcriptional and translational reprogramming are tightly controlled and can serve as adaptive responses to physiological stressors such as starvation to ensure selective protein expression ${ }^{12,13}$. During starvation, there is an initial decrease in global protein synthesis accompanied by the induction of alternative mechanisms for initiating the translation of mRNA that are followed by later translational recovery ${ }^{12} . \square$ Impaired translation also has been suggested to be involved in metabolic disorders such as $\mathrm{NASH}^{14,15}$. It is thought that induction of unfoldedprotein response (UPR)-mediated endoplasmic reticulum-associated degradation 
(ERAD) in response to ER stress and in conjunction with decreased overall protein translation, may be failed responses for maintaining hepatic cell homeostasis in $\mathrm{NASH}$. These changes then lead to the selective expression of inflammation and

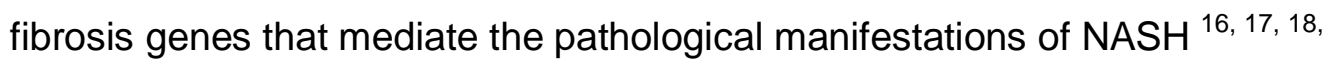

19. Interestingly, autophagy, a lysosome-dependent catabolic pathway that promotes protein degradation also is induced during starvation to maintain the amino-acid pool necessary to sustain essential protein translation. It also plays a critical role in mediating $\beta$-oxidation of fatty acids, and mitochondria and inflammasome clearance in the liver ${ }^{20,21}$. Of note, dysregulated autophagy has been associated with $\mathrm{NASH}^{18}$, 20, 22. Conversely, autophagy improves NASH pathogenesis and progression ${ }^{23,24,25}$, However, there have not been any reports thus far that have directly linked impaired protein translation during starvation to autophagy and its role in $\mathrm{NASH}$ pathogenesis.

In this report, we used cell culture and in vivo models to show decreased protein translation rate during acute starvation that was later followed by selective increases in protein translation rates. Unbiased proteomic analysis revealed that the expression of ribosome protein, Rplp1 and lysosome proteins, and Esrra-Ppargc1amediated signaling were increased during chronic starvation. Moreover, induction of Esrra was necessary for increased ribosomal protein expression, lysosomal protein translation and function, as well as autophagy during chronic starvation. Interestingly Esrra-Rplp1-dependent translation of lysosomal proteins and autophagy were impaired in livers from patients and mice with $\mathrm{NASH}$, and impeded the hepatic translational response to fasting in mice with $\mathrm{NASH}$. Both pharmacologic and genetic activation of the Esrra-Rplp1-lysosome pathway reduced NASH-related inflammation and fibrosis genes in cell culture and in vivo. Remarkably, the Esrra-Rplp1-lysosome 
pathway could be activated by alternate-day fasting in mice with NASH and improve NASH phenotype. In summary, we have discovered a novel Esrra-Rplp1-lysosome protein translation pathway linking protein translation to autophagy that is activated in chronic starvation and impaired in NASH.

\section{Results $\square$}

\section{Protein translation rate was temporally regulated during starvation}

To understand protein translation during acute and chronic starvation, primary human hepatocytes (PHHep) and mouse hepatic cells (AML12) were cultured in serum-free media (serum starvation) for $0,6,24,48$, and $72 \mathrm{~h}$ followed by puromycin (10 $\mu \mathrm{g} / \mathrm{ml}$ for $15 \mathrm{~min}$ ) incorporation. We then performed Western blotting on cell lysates to analyze puromycin-incorporated nascent proteins as a measure of protein translation rate ${ }^{26,27}$. There was a time-dependent decrease in puromycinincorporated nascent proteins from 6-48 h during serum starvation, followed by partial translational rate recovery at $72 \mathrm{~h}$ in both hepatic cell lines (Figure 1A-D).

\section{Temporal regulation of protein translation was selectively associated with ribosomal and lysosomal proteins during chronic starvation}

We then performed an unbiased label-free proteomic analysis in AML12 cells that underwent serum starvation for 0,48 and $72 \mathrm{~h}$ to characterize the proteins expressed at these time points. Surprisingly, despite the decrease in overall translation rate that we observed at $48 \mathrm{~h}$ (Figure 1A-D), approximately $10 \%$ of proteins detected in the total proteome (445/4082 proteins) showed increased expression (> 50\% higher expression than unstarved AML12 cell $(0 \mathrm{~h})$ ) at $48 \mathrm{~h}$ of serum starvation, and $20 \%$ of proteins in the total proteome (848/4066 proteins) 
were induced at $72 \mathrm{~h}$ of serum starvation (Figure 1E). We next analyzed all upregulated proteins at both time points for major associated pathways using the Kyoto Encyclopedia of Genes and Genomes (KEGG) 2019, and Gene Ontology Cellular Components (GO CC) 2018 databases (Figure 1F, Supplementary Figure 1A). Surprisingly, $55 \%$ of the total up-regulated proteins were induced at $72 \mathrm{~h}$ of serum starvation and enriched for pathways regulating translation, ribosomes, polysomes, RNA transport, ribosome biogenesis, autolysosomes, as well as lysosomes in the KEGG analysis (Figure 1E, F, Supplementary Figure 1A, Supplementary Tables 1-3). 14\% of the up-regulated proteins were exclusively induced at $48 \mathrm{~h}$ of starvation and did not correlate with any particular pathway (Figure 1F, Supplementary Table 4). However, when we combined them with the commonly upregulated proteins at $48 \mathrm{~h}$ of starvation, we identified pathways regulating chemical carcinogenesis, drug metabolism and proximal tubule bicarbonate reclamation-(Figure 1F, Supplementary Tables 1 and 5). Similar findings for pathways uniquely up-regulated at 48 and $72 \mathrm{~h}$ of starvation also were observed in GO CC analyses (Supplementary Tables 6-8). Interestingly, 31\% of the upregulated proteins were commonly induced at both 48 and $72 \mathrm{~h}$ of starvation. KEGG analyses of the commonly induced proteins revealed pathways related to lysosomes, fatty acid degradation, and mitochondrial oxidative phosphorylation (OXPHOS) (Figure 1F, Supplementary Figure 1A, Supplementary Table 1). Likewise, GO CC analysis also confirmed that mitochondria and lysosomes were among the most significantly upregulated cellular components induced at both 48 and $72 \mathrm{~h}$ of starvation (Supplementary Figure 1A;

\section{Supplementary Tables 6-8).}


Closer inspection of the protein expression heat maps showed that most of the proteins comprising the ribosome pathway were downregulated at $48 \mathrm{~h}$ of serum starvation compared to baseline, but rose to higher than baseline at $72 \mathrm{~h}$ starvation (Figure 1G). In particular, the large ribosomal subunit protein, Rplp1, was the most upregulated ribosomal protein at $72 \mathrm{~h}$ starvation. The expression of many of the lysosomal proteins were modestly upregulated at $48 \mathrm{~h}$ of serum starvation compared to their expression at baseline (Figure 1H) with the notable exceptions of Man2b1, Gla, and Gm2a which were down-regulated. Interestingly, expression of most of the lysosomal proteins, including Ctsd and Lamp2 as well as Man2b1, Gla, and $\mathrm{Gm} 2 \mathrm{a}$, were higher at $72 \mathrm{~h}$ of serum starvation than at $48 \mathrm{~h}$ (Figure $\mathbf{1 H}$ ). Additionally, expression of proteins corresponding to fatty acid degradation (e.g., Acox3, Acaa2, and Hadha) and OXPHOS (e.g., Ndufa9, Ndufb9, and Sdha) were modestly increased at $48 \mathrm{~h}$ of starvation and further increased at $72 \mathrm{~h}$

(Supplementary Figures 1B and 1C). To validate the proteomics data, we performed puromycin immunoprecipitation of puromycin-incorporated proteins at 48 and $72 \mathrm{~h}$ of serum starvation and found ribosomal protein Rplp1 was highly enriched at $72 \mathrm{~h}$ whereas lysosomal proteins Lamp2 and Ctsd were slightly enriched at $48 \mathrm{~h}$ serum starvation and further enriched at $72 \mathrm{~h}$ (Figure 1I).

\section{Esrra regulated ribosome-catalyzed translation of lysosome proteins during chronic starvation}

We next performed a transcription factor analysis of upregulated proteins and identified Ppargc1a and Esrra as the transcription factors most likely involved in the increases in protein translation at $72 \mathrm{~h}$ of serum starvation (Figure 1J). Estrogenrelated receptor alpha (Esrra) is a nuclear hormone receptor whose transcriptional 
activity is dependent upon heterodimerization with peroxisome proliferator-activated receptor gamma coactivator-1 alpha (Ppargc1a) ${ }^{28}$. While Esrra is recognized to regulate the transcription of mitochondrial genes, its role(s) in the regulation of ribosomes and lysosomal protein expression was not known. Accordingly, we performed Esrra siRNA knockdown (KD) in AML12 cells (Figures 1K and $\mathbf{1 L}$ ) and found that Esrra siRNA KD decreased Rplp1, Lamp2, and Ctsd protein expression. We then analyzed lysosomal activity by staining serum-starved Esrra KD cells with acridine orange $\mathrm{AO}$, and found they had decreased red fluorescence in control cells, consistent with decreased lysosomal activity-(Figures $1 \mathbf{M}$ and $\mathbf{1 N}$ ). We also showed that Esrra gene KD inhibited mitochondrial fatty acid fuel oxidation, respiratory activity, OXPHOS, and ATP production in AML12 cells (Supplementary Figures 1D-F). These findings are consistent with the cellular processes previously identified by the protein pathway analysis (Supplementary Figures 1B and 1C). Furthermore, Esrra or Rplp1 gene KD in AML12 cells inhibited overall puromycin incorporation and Lamp2 expression suggesting that reduced expression of either protein decreased translation rate and lysosomal protein expression (Figures 10-Q). In contrast, overexpression of either Esrra or Rplp1 in AML12 cells significantly increased puromycin-incorporated proteins as well as Lamp2 protein expression (Figures 1RT). The co-regulation of Lamp2 by either Rplp1 or Esrra expression in these experiments suggested that Esrra may regulate Rplp1 expression and ribosomecatalyzed protein translation to increase lysosomal proteins during chronic starvation.

\section{Expression of autophagy proteins were temporally regulated during chronic} serum starvation and associated with Esrra-Rplp1 axis 
Starvation induces autophagy via a lysosome-dependent catabolic pathway ${ }^{29,30}$. Accordingly, we examined the temporal regulation of autophagyrelated proteins, Map1lc3b-ii and Sqstm1 as well as Esrra, Rplp1, Lamp2 and Ctsd at baseline, $24 \mathrm{~h}, 48 \mathrm{~h}$, and $72 \mathrm{~h}$ of serum starvation (Figure 2A-C). Map1lc3b-ii protein expression increased at $24 \mathrm{~h}$, decreased at $48 \mathrm{~h}$, and then increased at $72 \mathrm{~h}$ of serum starvation. Interestingly, Sqstm1 expression decreased at $24 \mathrm{~h}$, increased at $48 \mathrm{~h}$, and decreased at $72 \mathrm{~h}$ suggesting there was an autophagy increase at $24 \mathrm{~h}$, a late block in autophagy at $48 \mathrm{~h}$, and a recovery of autophagy at $72 \mathrm{~h}$. Esrra protein expression increased at $24 \mathrm{~h}$, peaked at $48 \mathrm{~h}$, and remained elevated at $72 \mathrm{~h}$ (Figure 2B). The temporal expression pattern of Map1lc3b-ii protein closely matched those of Rplp1, Lamp2, and Ctsd proteins (Figure 2C), and suggested that decreased lysosome protein synthesis could be involved in the late block in autophagy at $48 \mathrm{~h}$ and increased lysosome protein synthesis in the recovery of autophagy at $72 \mathrm{~h}$.

We next examined the translation rates of autophagy proteins during serum starvation by analyzing puromycin-incorporated proteins (Figure 2D). We observed a slight increase in puromycin-incorporated Map1lc3b-ii at $48 \mathrm{~h}$ serum starvation that was markedly increased at $72 \mathrm{~h}$. Several other autophagy-related proteins, Sqstm1 and Atg5 as well as Med1 (a co-activator protein involved in stimulating autophagy) ${ }^{31}$ had a similar temporal puromycin incorporation patterns; however, Atg7 puromycin incorporation increased at $48 \mathrm{~h}$ and did not change between 48 and $72 \mathrm{~h}$ serum starvation. Significantly, proteomic analysis also revealed that Map1lc3b, Sqstm1, Atg5, and Med1 increased at $72 \mathrm{~h}$ of serum starvation compared to $48 \mathrm{~h}$ whereas Atg7 remained the same at $72 \mathrm{~h}$ (Supplementary Figure 2A). Thus, our data showed that induction of Esrra protein expression preceded the increases in Rplp1, 
Lamp2, Ctsd, Map1lc2b-ii, and Sqstm1 protein expression and led to marked increases in protein translation rates of lysosome and autophagy proteins at $72 \mathrm{~h}$ of serum starvation.

To confirm Esrra regulated autophagic flux during starvation, we transiently expressed RFP-eGFP-Map1lc3 plasmid in control and Esrra siRNA-treated AML12 cells and then subjected them to serum starvation (Figure 2E, Supplementary Figures 2B and 2C). We observed increased red puncta formation in control cells (Figure 2E, Supplementary Figures 2B) indicating that there was an increase in autophagy flux since GFP fluorescence was quenched in active lysosomes. In contrast, Esrra siRNA-treated cells exhibited markedly increased yellow puncta formation in media with serum and even more in serum-free media. The yellow puncta arose from increased fluorescence of both GFP and RFP due to inhibition of autophagy flux (Figure 2E, Supplementary Figure 2C). Using a lysosomal inhibitor, Bafilomycin A to analyze starvation-induced autophagy flux, we found there was less accumulation of Map1lc3b-ii during starvation-induced autophagy flux in Esrra KD AML12 cells than control cells (Figures $\mathbf{2 F}$ and $\mathbf{2 G}$ ), and more accumulation in Esrra-overexpressed cells than control cells (Figure $\mathbf{2 H}$ and $\mathbf{2 l}$ ), confirming that Esrra expression regulated autophagy flux.

\section{Esrra, Rplp1, lysosome and autophagy proteins were co-regulated in vivo during starvation}

To better understand Esrra's regulation of ribosome-dependent protein translation and its effects on lysosome-autophagy function in vivo, we analyzed liver tissues from wild-type (WT) and Esrra knockout (KO) mice starved for $24 \mathrm{~h}$ and compared them with fed controls (Figure 3A and 3B). Esrra KO mice in the fed state 
showed a significant decrease in Rplp1 expression and a downward trend in the expression of Lamp2 and Map1lc3b-ii proteins compared to WT mice. Starvation increased Esrra, Rplp1, Lamp2, Ctsd, and Map1lc3b-ii protein expression, and had no significant effect on Sqstm1 protein in WT mice. In Esrra KO mice, these increases in the protein expression during starvation were significantly attenuated, and Sqstm1 expression increased during starvation suggesting that autophagy was inhibited when Esrra was not expressed (Figure 3A and 3B). Moreover, the inverse agonist of Esrra, XCT790 significantly decreased Esrra, Rplp1, and Lamp2 protein expression in WT mice (Supplementary Figures 3A and 3B). Hepatic lysosome number also was significantly decreased in XCT790-treated mice compared to vehicle-treated controls suggesting that Esrra regulated lysosome number (Supplementary Figures 3C and 3D). Hepatic Esrra overexpression in mice increased expression of Rplp1, Lamp2, and Ctsd proteins and promoted autophagy flux as evident by increased Map1lc3b-ii and decreased Sqstm1 expression (Figures 3C and 3D).

To better understand Esrra's effects on translation rate during starvation in vivo, we injected mice with vehicle (control) or a small molecule inhibitor of Esrra, C29 during starvation, and measured puromycin incorporation at baseline, 8, 24, and 48 h starvation. Protein translation rate decreased from 8 to $24 \mathrm{~h}$ and significantly increased at $48 \mathrm{~h}$ compared to $24 \mathrm{~h}$ in livers from control mice suggesting some recovery at $48 \mathrm{~h}$ (Figures $3 \mathrm{E}$ and $\mathbf{3 F}$ ). In contrast, there was a significant inhibition of overall puromycin incorporation from 8 to 48 h starvation in C29-treated mice compared to their fed state (0 h) (Figures 3E and 3F). Esrra, Lamp2, Ctsd, and Map1lc3b-ii proteins expression progressively increased from $8 \mathrm{~h}$ to $48 \mathrm{~h}$ starvation in vehicle-treated mice (Figures 3E, G-J). However, the expression of Esrra, Rplp1, 
Lamp2, and Ctsd proteins was significantly lower in C29-treated mice than vehicletreated mice at each time point. Map1lc3b-ii expression also was inhibited and Sqstm1 expression was increased in C29-treated mice at $24 \mathrm{~h}$ and $48 \mathrm{~h}$ starvation (Figures 3E, G-J). We also observed similar findings in C29-treated AML12 cells during acute and chronic serum starvation (Supplementary Figures 3E-G) by measuring puromycin incorporation in C29-treated and untreated AML12 cells at 48 and $72 \mathrm{~h}$ starvation. We observed significant decreases in protein translation rates for both groups after $48 \mathrm{~h}$ serum starvation; however, untreated control cells resumed translation at $72 \mathrm{~h}$ serum starvation whereas C29 treatment of AML12 cells had markedly decreased translation rate at $72 \mathrm{~h}$ starvation (Supplementary Figures 3E-G). Esrra, Rplp1, Lamp2, Ctsd proteins expression increased in control cells at $72 \mathrm{~h}$ whereas they were not changed or decreased in C29-treated cells.

In parallel with the changes in protein translation rate, we observed significant time-dependent increases in Esrra, Rplp1, Lamp2, Ctsd, Map1/c3b-ii and Sqstm1 gene expression in WT mice and AML12 cells undergoing starvation (Supplementary Figures $\mathbf{3 H}$ and 3I). Interestingly, although $\mathrm{C} 29$ treatment of mice and AML12 cells significantly inhibited Esrra and Rplp1 gene expressions during starvation, Lamp2, Ctsd, Map1/c3b-ii and Sqstm1 mRNA expressions were significantly higher than controls perhaps due to compensatory mechanism(s) for decreased protein translation rate. The progressive increases in Esrra and Rplp1 mRNAs correlated with their increased protein translation rates in both control mice and AML12 cells; however, Esrra and Rplp1 mRNA expressions were downregulated or unchanged at $48 \mathrm{~h}$ in C29-treated mice and at $72 \mathrm{~h}$ in AML12 cells (Supplementary Figures $\mathbf{3 H}$ and $3 \mathrm{I}$ ). These findings suggest that Esrra and Rplp1 protein expression were transcriptionally regulated by Esrra whereas 
Lamp2, Ctsd, Map1/c3b-ii and Sqstm1 transcription was not dependent upon Esrra, and their protein expression was primarily translationally-regulated by Rplp1 expression. In support of this notion, we discovered a strong Esrra binding site on the Rplp1 promoter in our previously published mouse liver Esrra ChIP-seq database 2832 (Supplementary Figure 3J). Esrra is known to autoregulate positively its own transcription ${ }^{33} 34$ and there was strong Esrra binding to its own promoter. Surprisingly, we also found weak Esrra binding on the Lamp2, Ctsd, Map1/c3bii and Sqstm1 gene promoters (Supplementary Figure 3J). Taken together, our findings showed that Esrra regulated the translation rate of lysosome-autophagy proteins in hepatic cells and mouse liver during chronic starvation, most likely via transcriptional induction of the ribosomal large subunit protein Rplp1 by Esrra.

\section{Decreased $\beta$-oxidation of fatty acids and increased hepatosteatosis in mice treated with C29 undergoing starvation}

We next examined the role of Esrra on the liver during fasting. Surprisingly, normal chow diet fed mice injected with C29 for three days had significant increases in body weight and liver index compared to vehicle-treated control mice at baseline (Supplementary Figures 4A and 4B). Control mice had significant increases in serum $\beta$-hydroxybutyrate $(\beta-H B)$ in a time= dependent manner with no significant change in liver triglyceride (TG) levels and liver index in control mice during starvation (Supplementary Figures 4C-E). In contrast, C29-treated mice had an attenuated serum $\beta$-HB increase during $48 \mathrm{~h}$ starvation. Their liver index and liver TG levels also increased and were higher than control mice during starvation (Supplementary Figures 4C-E). These findings showed that Esrra inhibition 
led to decreased induction of hepatic $\beta$-oxidation of fatty acids and accumulation of hepatic triglycerides during starvation. These physiological changes also were accompanied by decreased Rplp1-mediated translation of lysosomal proteins and autophagy (Figures 3E-J). It is noteworthy that decreased autophagy previously was reported to reduce $\beta$-oxidation of fatty acids and cause hepatosteatosis $20,23,25,33$.

\section{Esrra-Rplp1-lysosome translation pathway was impaired in patients and mice $\square$ with NASH}

Currently, little is known about the effects of starvation on the regulation of translation in NAFLD and its more advanced stage, NASH. Thus, we wondered whether there could be a defect in the Esrra-Rplp1 axis in these conditions. Accordingly, we analyzed liver tissues collected from a small cohort of patients (Department of Surgery, Singapore General Hospital) with steatosis and NASH, and controls ( $\mathrm{n}=5$ per group); and found increased expression of inflammatory (TNFA, IL1B, IL6, CCL2 and CCL5) and fibrosis (TGFB, COL1A1,COL1A2, COL3A1, ACTA2 and MMP9) marker genes in steatosis samples, that became significantly higher in NASH samples compared to controls (Supplementary Figure 4F). These findings confirmed their pathological categorization for hepatosteatosis and NASH. Remarkably, the protein expressions of ESRRA, RPLP1, LAMP2, and CTSD also were significantly decreased in steatosis and further reduced in NASH (Figures 4A and 4B) suggesting that ESRRA-mediated regulation of lysosomal protein expression could be impaired. 
To address this issue, we examined a dietary mouse model of progressive $\mathrm{NASH}$ in which mice were fed a Western diet with $15 \% \mathrm{w} / \mathrm{v}$ fructose in drinking

water (WDF) for 8 and 16 weeks to induce steatosis and NASH, respectively ${ }^{35}$. Hepatic inflammatory and fibrosis gene expression was significantly increased in steatosis at 8 weeks and further increased in NASH at 16 weeks (Supplementary Figure 4G). Histological analysis using Hematoxylin and Eosin (H\&E) staining further confirmed progressive NASH pathogenesis i.e. micro/macro steatosis and inflammatory foci in WDF 16 weeks (Figures 4C). Further, similar to our clinical observations, hepatic Esrra, Rplp1, Lamp2, and Ctsd protein expression were progressively decreased during steatosis and NASH in mice fed WDF compared to control mice fed NCD (Figures 4D and 4E).

\section{Protein translation rate and autophagy $\square$ decreased when Esrra-Rplp1-} lysosome translation pathway was impaired in NASH

To determine further whether the decreases in Esrra, Rplp1, Lamp2, and Ctsd protein expression in NASH were due to decreased translation, we fed mice with methionine-choline deficient (MCD) diet for 3 and 6 weeks to rapidly generate $\mathrm{NASH}^{36,37}$. These mice exhibited progressive increases in inflammatory and fibrosis marker gene expression, serum $\beta-\mathrm{HB}$, and liver TG, at 3 and 6 weeks without any change in serum TG (Supplementary Figures $\mathbf{4 H - 4 J )}$ ). H\&E staining also confirmed progressive NASH pathogenesis from 3 and 6 weeks of MCD diet feeding i.e. steatosis, inflammatory foci and fibrotic regions (Figures 4C). Morover, we observed progressive decreases in puromycin-incorporated proteins in mice fed MCD diet at 3 and 6 weeks 
(Figures $\mathbf{4 G}$ and $\mathbf{4 H}$ ), suggesting that protein translation rate decreased as NASH progressed. Furthermore, expression of Esrra, Rplp1, Lamp2, Ctsd and Map1lc3b-ii proteins decreased progressively whereas Sqstm1 protein increased in livers from mice fed MCD diet suggesting there was decreased autophagy flux and translation rates of lysosomal proteins

(Figures 4G and 4I). To further demonstrate this Esrra-Rplp1-lysosome translation pathway in hepatic cells, we treated PHHep and AML12 cells with palmitic acid (PA) to induce lipotoxicity $25,36,37,38,39$. We found increased expression of inflammation and fibrosis genes and Casp3 cleavage (a hallmark of lipotoxicity in hepatocytes ${ }^{38}$, and decreased expression of Esrra, Rplp1, Lamp2, Ctsd, Map1lc3b-ii in PA-treated cells (Figures 4J-O, Supplementary Figure $4 \mathrm{~K}$ ). These findings showed that lipotoxic conditions were sufficient to down-regulate the Esrra-Rplp1-lysosome translation pathway and autophagy, and confirmed our in vivo NASH findings.

\section{Translation dynamics and Esrra-Rplp1-lysosome pathway were deregulated} during starvation in NASH $\square$

We next examined whether the Esrra-Rplp1-lysosome translation pathway was altered during starvation in NASH by employing a dietary model of $\mathrm{NASH}$, in which we fed mice WDF for 16 weeks before starving them for 0, 24 and $48 \mathrm{~h}$, and injecting them with puromycin (Puro, $20 \mathrm{mg} / \mathrm{kg}$ body weight) i.p. $30 \mathrm{~min}$ before euthanization. Consistent with our findings in mice fed MCD (Figures $\mathbf{4 G}$ and $\mathbf{4 H}$ ), mice fed WDF had reduced total puromycin incorporation and decreased Esrra, Rplp1, Lamp2, Ctsd, and Map1lc3v-ii protein translation rates at baseline compared to mice fed NCD (Supplementary Figures 5A and 5B). Mice fed NCD which had increased 
overall protein translation rate at 48 hours of starvation (Figures $3 E, 3 F$ ). In contrast, mice fed WDF did not have any significant increase in overall protein translation rate at $48 \mathrm{~h}$. Moreover, Esrra, Rplp1, Lamp2, Ctsd, and Map1lc3b-ii protein translation rates did not increase relative to baseline levels at $48 \mathrm{~h}$ of starvation in mice fed WDF whereas they increased during starvation in mice fed NCD (Figures 3E and 3F; Supplementary Figures 5A, and 5C). Sqstm1 protein accumulated in mice fed WDF at $48 \mathrm{~h}$ of starvation, suggesting there was decreased autophagy flux (Supplementary Figures 5A and 5C). In contrast, mice fed NCD had increased autophagy flux at $48 \mathrm{~h}$ of starvation (Figures 3E and 3J). Moreover, Lamp2, Ctsd, Map1/c3b and Sqstm1 gene expressions were significantly higher at 48h starvation in livers from mice fed WDF (Supplementary Figure 5D), most likely as compensatory mechanism(s) for reduced autophagy. Taken together, these findings showed that mice with diet-induced NASH had dysregulated translation, inhibited Esrra-Rplp1-lysosome pathway and blocked autophagy during $48 \mathrm{~h}$ starvation while transcription of lysosome genes and Map1lc3b remained intact.

The reduced induction of the Esrra-Rplp1-lysosome pathway in NASH also had physiological consequences during starvation. When mice fed WDF for 16 weeks were starved for 24 and 48 h (Supplementary Figures 5A-D), their liver index decreased at $24 \mathrm{~h}$ and remained unchanged at $48 \mathrm{~h}$ of starvation (Supplementary Figure 5E). Serum $\beta-\mathrm{HB}$ increased at $24 \mathrm{~h}$ of starvation in mice fed WDF but in contrast to control mice fed NCD, it did not further increase at $48 \mathrm{~h}$ starvation (Supplementary Figures 4C and 5F). Hepatic TG of mice fed WDF was elevated relative to hepatic TG of control mice fed NCD, and remained unchanged during $48 \mathrm{~h}$ of starvation (Supplementary Figure 5G). 


\section{Activating Esrra-Rplp1-lysosome translation pathway improved NASH in vitro and in vivo}

We next examined whether activating Esrra-Rplp1-lysosome translation pathway could be a potential therapeutic strategy for treating NASH. Accordingly, we overexpressed Esrra and Rplp1 in AML12 cells treated with PA. Interestingly, both Esrra or Rplp1 overexpression increased overall puromycin-incorporated protein expression that was decreased by PA treatment (Figures 5A and 5B), suggesting that activating Esrra or Rplp1 could stimulate ribosome-dependent protein translation in PA-treated cells. Esrra or Rplp1 overexpression also decreased Casp3 cleavage in PA-treated AML12 cells as well as increased the translation rates of Lamp2, Ctsd, Map1lc3b-ii, and Sqstm1 proteins suggesting there was improved lysosomal activity and autophagy (Figures 5A and 5C). Additionally, the induction of inflammatory (Tnfa, I/1b, I/6, Ccl2, and Ccl5) and fibrosis (Tgfb, Col1a1, Col1a2, Col3a1, Acta2, and Mmp9) gene expression by PA was attenuated by Esrra and Rplp1 overexpression (Supplementary Figure 5H). We further examined whether a putative Esrra activator, genistein ${ }^{33,40,41}$ could restore the Esrra-Rplp1-lysosome translation pathway in PA-treated hepatic cells. PHHep cells treated with PA and genistein (Supplementary Figures 5I-K) showed recovery of puromycinincorporated protein, increased translation rates of ESRRA, RPLP1, LAMP2, CTSD, MAP1LC3B-II, and SQSTM1 proteins, improved autophagy, and decreased Casp3 cleavage compared to PHHep cells treated with PA alone. We confirmed genistein's effects were Esrra-mediated since they were blocked by C29 co-treatment (Supplemental Figures 5I and 5K). 
We next examined hepatic Esrra overexpression in mice fed WDF and found that it significantly increased hepatic Rplp1, Lamp2, Ctsd, and Map1lc3b-ii protein expression compared to control mice fed WDF (Figures 5D and 5E). Interestingly, the Sqstm1 protein accumulation in control mice fed WDF (suggesting late block autophagy) decreased in hepatic Esrra-overexpressing mice fed WDF, and when combined with an increase in Map1lc3b-ii expression, suggest there was improved autophagy flux in these Esrra-overexpressing WDF-fed mice. Furthermore, hepatic Esrra overexpression in mice fed WDF had reduced liver index, fasting liver TG, fasting serum glucose and increased fasting serum $\beta-\mathrm{HB}$ compared to control mice fed WDF (Figures 5F-I). Esrra-overexpressing mice fed WDF had reduced hepatic inflammation and fibrosis gene expression compared to control mice fed WDF suggesting improvement in NASH (Figure 5J). Histological analysis of H\&E staining also confirmed that there were improvements in NASH pathological features such as micro/macro steatosis and inflammatory foci in Esrra-overexpressing WDF fed mice when compared to Null-WDF mice (Figures 5K).

\section{Alternate day fasting-improved NASH required activation of translation and Esrra-Rplp1-lysosome pathway}

Intermittent fasting regimens such as alternate day fasting for several days have had beneficial effects in obesity, diabetes as well as in NASH ${ }^{42}$. Since Esrra protein was induced during fasting and hepatic Esrra overexpression in mice fed WDF activated the Esrra-Rplp1-lysosome pathway to improve autophagy and reduce hepatosteatosis, inflammation, and fibrosis, we examined whether intermittent fasting could induce the Esrra-Rplp1-lysosome pathway and have beneficial effects on NASH. Accordingly, we employed an alternate day fasting regimen for two weeks 
in mice fed WDF for 16 weeks to pre-establish NASH. These mice with NASH then were injected with vehicle or C29 during fasting to examine the role of Esrra in intermittent fasting (Figure 6A). Mice fed NCD or WDF continuously were used as controls. Similar to the mice fed MCD (Figures 4E-F), mice fed WDF for 16 weeks developed NASH and had significantly reduced puromycin-incorporation of overall proteins and of Esrra, Rplp1, Lamp2, Ctsd and Maplc3b-ii and Sqstm1 suggesting there was impaired lysosome-autophagy protein translation and function at baseline (Figures 6B-E). Surprisingly, alternate day fasting for two weeks reversed the decreases in puromycin-incorporated proteins in mice fed WDF, and restored Esrra, Rplp1, Lamp2, Ctsd, Map1lc3b-ii and decreased Sqstm1 protein expression suggesting there was improved autophagy (Figures 6B-E). In contrast, C29 injection during fasting significantly inhibited these changes and increased Sqstm1 expression suggesting impaired autophagy. Remarkably, mice fed WDF that underwent alternate day fasting had significant improvements in serum $\beta-\mathrm{HB}$, liver index, and serum glucose levels in conjunction with increased Esrra protein expression (Figure 6F, 6G, Supplementary Figure 6A). In contrast, mice fed WDF that underwent alternate day fasting and C29 injection during alternate day fasting had little or no improvement in these parameters compared to mice fed WDF continuously. Interestingly, hepatic TG content in mice fed WDF that underwent alternate day fasting remained the same as mice fed WDF continuously (Supplementary Figure 6B). Additionally, mice fed WDF undergoing alternate day fasting had reduced expression of inflammatory and fibrosis genes compared to mice fed WDF continuously or mice fed WDF and injected with C29 while undergoing intermittent daily fasting (Figures 6H). H\&E staining also showed that alternate day fasting improved NASH pathological features (micro/macro steatosis 
and inflammatory foci) in WDF fed mice, while C29 treatment worsened these features (Figures 6I).

\section{Discussion}

$\square$ During acute fasting, nutrient-sensing pathways cause a rapid down-regulation of anabolic pathways, including global protein synthesis ${ }^{30,43}$. These changes are accompanied by the induction of alternative mechanisms for initiating the translation of existing mRNA and followed by a translational recovery ${ }^{12}$. Despite these observations, little is known currently about the regulation of protein translation during acute and prolonged starvation. Thus, we investigated the hepatic changes in translation during starvation since the liver undergoes major metabolic changes during starvation and is the key manager of nutrient supply to other organs during starvation. Initially, we found that general protein translation rate was down-regulated acutely in hepatic cells in cell culture and in vivo and was followed by partial recovery of general protein translation rate. We then performed proteome analysis of hepatic cells undergoing starvation for $48 \mathrm{~h}$ and $72 \mathrm{~h}$, and found induction of proteins regulating ribosomal, lysosomal, and mitochondrial pathways. Surprisingly, further examination revealed that increased Ppargc1a-Esrra expression led to transcriptional induction of Rplp1, which then catalyzed translational recovery of several lysosomal and mitochondrial proteins during starvation. Furthermore, we identified an Esrra-binding site on the promoter of the Rplp1 gene and Esrra KD decreased ribosomal protein Rplp1 mRNA and protein expression. In contrast, Esrra KD had no effect on mRNA expression but decreased translation of Lamp2 and Ctsd lysosomal proteins in a Rplp1-dependent manner. Our findings were further 
supported by previous studies in Ppargc1a knockout (KO) mice which had decreased Lamp2 and Ctsd expression in vascular smooth muscle cells ${ }^{44}$.

Starvation is known to induce autophagy; however, the mechanism for this response is poorly understood ${ }^{29,30}$. Here, we observed temporal regulation of the translation of the autophagy-related proteins, Map1lc3b-ii and Sqstm1, in coordination with Esrra, Rplp1, Lamp2 and Ctsd expression during $72 \mathrm{~h}$ of serum starvation. Map1lc3b-ii and Sqstm1 protein levels suggested there was an initial later block in autophagy at $24 \mathrm{~h}$ of starvation that was restored after the increases in the Rplp1, Lamp2, and Ctsd protein translation occurring at $72 \mathrm{~h}$ of starvation. In addition to the translational recovery of lysosomal proteins, we observed that the protein expression of Med1, a nuclear receptor co-activator protein that has recently was shown to be an important coactivator for autophagy genes ${ }^{31}$, was induced at 48 and $72 \mathrm{~h}$ suggesting that there also may be a transcriptional contribution to the recovery of autophagy during late starvation. We previously showed that Esrra regulated hepatic mitochondrial activity ${ }^{28}$. Interestingly, we found that mitochondrial protein expression and activities (oxidative phosphorylation and $\beta$-oxidation) correlated with Esrra expression during starvation in cell culture and in vivo.

The effects of NASH on protein translation are poorly understood. Surprisingly, we found that the general protein translation rate, the Esrra-Rplp1-lysosome pathway and autophagy flux were impaired in NASH (Supplementary Figure 7). Moreover, the general translational recovery and the improvement in lysosomal protein translation rate and autophagy during prolonged starvation were hindered in mice with NASH. These, in turn, led to decreased $\beta$-oxidation of fatty acids and higher hepatic triglyceride content during both the fed and starved states in mice with 
$\mathrm{NASH}$. It is possible that activation of the Esrra-Rplp1-lysosome pathway may enable hepatic cells to withstand stresses such as insulin-resistance, starvation, lipotoxicity, oxidative stress, and inflammation during NASH since its stimulation of autophagy reduces hepatic triglyceride content (via lipophagy) and inflammasome formation, and removes and degrades damaged mitochondria (via mitophagy). Indeed, over-expression of Esrra improved lysosome-autophagy function and led to decreased expression of inflammation and fibrosis genes, and improved histological features in our dietary model of NASH.

We also showed that the Esrra-RIplp1-lysosome pathway can be activated pharmacologically by an Esrra agonist genistein, and metabolically by alternate day fasting. Indeed, we demonstrated that the beneficial effects of alternate day fasting on inflammation and fibrosis gene expression and histology in mice with NASH could be abrogated by co-administration of C29, an Esrra antagonist. Our findings thus provide a potential molecular explanation for some of the beneficial effects of alternate day fasting that have been reported in patients with $\mathrm{NASH}^{7,42,45,46}$. Our results also point to a central role of Esrra in NASH progression and recovery, and suggest that the Esrra-Rplp1-lysosome translation pathway could be a promising new therapeutic target for NASH. Remarkably, our data showed that nuclear hormone receptors such as Esrra, which are known to regulate transcription, can regulate translation under certain circumstances such as starvation and $\mathrm{NASH}$. Thus, it is possible that compounds which induce Esrra expression or act as Esrra agonists could activate the Esrra-Rlplp1-lysosome translation pathway and reduce NASH progression in combination or in lieu of alternate day fasting. In this connection, we previously showed that TH can induce Ppargc1a and Esrra expression to increase hepatic autophagy and mitochondrial activity ${ }^{28}$, and thus 
could activate this protein translation pathway, in addition to its transcriptional effects on autophagy genes, to reduce NASH progression. Currently, it is not known whether the Esrra-RIplp1-lysosome translation pathway is dysregulated in other hepatic, metabolic, or oncologic conditions. Interestingly, Esrra has been shown to positively regulate autophagy in several different cell types ${ }^{28,33,47}$. Pharmacologic inhibition of Esrra decreased autophagy to enhance neuroprotection or decrease cancer cell proliferation ${ }^{48,49,50}$. Future studies will help determine whether the perturbations in starvation response, autophagy, and protein translation due to decreased Esrra expression are unique to NASH or also occur in other pathological conditions.

\section{Methods (2346w/3000)}

Liver samples from human subjects and ethics statement $\square \square$

Histologically characterized liver biopsy samples were collected from the tissue repository Centre, Singapore General Hospital (SGH) under the protocol IRB 2016/2836, 2019/2119 and NUS-IRB-2020-206 and subsequently analyzed for mRNA and protein expressions.

\section{Animal studies $\square$}

MCD diet: 8-10 weeks old male C57BL/6J mice were fed with normal chow control diet (NCD) or methionine-and choline-deficient L-amino acid diet (MCD; A02082002BR, Research Diets, Inc.) for 3 and 6 weeks for progressively develop $\mathrm{NASH}^{37,51}$. Puromycin (Puro, $20 \mathrm{mg} / \mathrm{kg}$ body weight) was injected i.p. $30 \mathrm{~min}$ before euthanization for rate of translation analysis and cycloheximide $(\mathrm{CHX}, 0.1$ $\mathrm{mg} / \mathrm{g}$ body weight) was injected i.p. $60 \mathrm{~min}$ before euthanization to inhibit 
translation ${ }^{52,53}$. Later, mice were fasted for $6 \mathrm{~h}$ and euthanized, blood and liver tissues were collected for mRNA and protein analysis.

WDF diet: 8-10 weeks old male C57BL/6J mice were fed with normal chow control diet (NCD) or Western diet (WD; D12079B, Research Diets, Inc.) supplemented with 15\% (w/v) fructose (F0127, Sigma-Aldrich) in drinking water (WDF) for 8 or 16 weeks to progressively generate steatosis and NASH respectively $35,38,51$. Later, mice were fasted for $6 \mathrm{~h}$ and euthanized, blood and liver tissues were collected for mRNA and protein analysis.

Starvation study: 8-10 weeks old male C57BL/6J mice were fed with normal chow control diet (NCD) and starved overnight before starting the experiment for synchronization. After one day, mice were kept in new empty cages (without husk bedding) for $8,24,48 \mathrm{~h}$. C29 (10 mg/kg body weight) was started injecting i.p. from the synchronization day to $48 \mathrm{~h}$ fasting day for the chronic inhibition of Esrra ${ }^{54}$. Puromycin (Puro, $20 \mathrm{mg} / \mathrm{kg}$ body weight) was injected i.p. $30 \mathrm{~min}$ before euthanization for rate of translation analysis. Later, mice were euthanized, blood and liver tissues were collected for mRNA and protein analysis.

Alternate day fasting study: Mice were fed WDF for 16 weeks to induce NASH or NCD as control. After 16 weeks, mice were fasted and fed alternate days (Fast-Fed one cycle) for five cycle ( $n=4-5$ per group). For inhibiting Esrra specifically during fasting, we injected C29 (10 mg/kg body weight) or vehicle i.p. twice a day (5 h before the start and just before the start of each fasting cycle) in only fasting groups during fasting day while there was no injection given during fed days. Puromycin (Puro, $20 \mathrm{mg} / \mathrm{kg}$ body weight) was injected i.p. $30 \mathrm{~min}$ before euthanization for rate 
of translation analysis. Mice were euthanized during fed condition, blood and liver tissues were collected for mRNA and protein analysis.

Esrra KO mice: Esrra KO mice are described elsewhere ${ }^{54,55}$. Esrra WT and Esrra KO mice were synchronized by overnight fasting and starved for $24 \mathrm{~h}$ after two days or left fed. Later, mice were euthanized, blood and liver tissues were collected for mRNA and protein analysis.

Alb-mESRRA overexpression: For liver-specific expression, we used AAV8 mediated gene delivery of mEsrra gene cloned under the control of mouse Alb promoter (Vector Biolabs, USA). 8 weeks old male C57BL/6J mice were used for liver-specific Esrra overexpression (Alb-mEsrra). Mice were generated by injecting AAV8-Alb-mEsrra (5X1011 gc/mice) via tail vein and housed for four weeks with no other intervention ${ }^{38}$. We injected AAV8-Alb-Null which does not contain any DNA sequence under the transcriptional control of the Alb promoter as controls. Later, mice were euthanized, blood and liver tissues were collected for mRNA and protein analysis.

General mouse care and ethics statement: mice were purchased from InVivos, Singapore and, housed in hanging polycarbonate cages under a $12 \square \mathrm{h} / 12 \square \mathrm{h}$ light/dark schedule at Duke-NUS vivarium. Mice were fed different diets and normal water, or fructose treated ad libitum. Animals were euthanized in $\mathrm{CO} 2$ chambers. All mice were maintained according to the Guide for the Care and Use of Laboratory Animals (NIH publication no. One.0.0. Revised 2011), and the experiments performed were approved by the IACUCs at SingHealth (2015/SHS/1104) and (2020/SHS/1549). 
Blood glucose, serum $\beta$-hydroxybutyrate ( $\beta$-HB/Ketone bodies), and serum and liver triglycerides (TG) measurements.

Blood glucose was measured from euthanized mice during the blood collection using Roche's strip-based glucometer (Accu-Chek Performa, Roche). Serum_ $\beta$-HB and TG were measured using $\beta$-HB (Ketone Body) Colorimetric Assay Kit (\#700190, Cayman) and Triglyceride Colorimetric Assay Kit (\#10010303, Cayman).

Cell cultures $\square \square$

$\underline{\text { AML12 cells }}\left(\right.$ ATCC® $C R L-2254^{T M}$ ) were cultured as indicated elsewhere ${ }^{56,57}$. Starvation medium (DMEM:F12 mix with Pen/Strep lacking serum, ITS and Dexamethasone) was used for serum starvation experiments. To analyze the rate of translation, puromycin $(10 \mu \mathrm{g} / \mathrm{ml})$ was added for $15 \mathrm{~min}$ before harvest ${ }^{26}$ whereas $\mathrm{C} 29(5 \mu \mathrm{M})$ was added to media for indicated time to inhibit Esrra ${ }^{54}$. Palmitic acid (PA) $0.5 \mathrm{mM}$ conjugated with $0.5 \%$ BSA was used for $24 \mathrm{~h}$ as described elsewhere ${ }^{38}$. $0.5 \%$ BSA solution was used as control (CT). Bafilomycin A1 (5 nM) was used to analyze autophagy flux ${ }^{28}$.

Primary human hepatocytes (5200, ScienCell) were cultured as indicated elsewhere ${ }^{38,58}$. Puromycin $(10 \mu \mathrm{g} / \mathrm{ml})$ was added for $15 \mathrm{~min}$ before harvest ${ }^{26}$ whereas C29 $(5 \mu \mathrm{M})$ was added to media for indicated time ${ }^{54}$. PA was used as stated above. Genistein $(5 \mu \mathrm{M})$ for $24 \mathrm{~h}$ was used to activate Esrra.

Gene manipulation in cultured cells $\square$

Gene knockdown in vitro: Silencer Select siRNAs (s4829, s4830, and s4831; Life Technologies Inc.) or ON-TARGETplus Smartpool siRNAs (L-040772-00- 
0010, Dharmacon) against Esrra, and Silencer Select siRNA (s234520; Life Technologies Inc.) for Rplp1 gene knockdown were used in AML12 cells. Negative siRNA (Silencer Negative Control No. 1 siRNA; AM4611, Life Technologies Inc.) was used as a negative control. Transfections were carried out in AML12 cells in a 12well or 6-well plate or four-well chambered slides or 24-well Seahorse XF plate using $30 \mathrm{nM}$ of the above indicated siRNAs and negative control siRNA with Lipofectamine RNAiMAX (Invitrogen; Life Technologies Inc.) following the reverse transfection protocol, as indicated elsewhere ${ }^{28,56} . \square$

Gene overexpression in vitro: ORF sequence of mEsrra (Gene ID: 26379) and mRplp1 (Gene ID: 56040) genes were cloned in a pcDNA3.1(+)-C-6His plasmid by Genescript Limited (Hong Kong) to get Esrra_OMu13026C_pcDNA3.1(+)-C-6His and Rplp1_OMu11118C_pcDNA3.1(+)-C-6His constructs for Esrra and Rplp1 overexpression in AML12 cells respectively. Transfections were carried out in a 12-well plate or 6-well plate using Lipofectamine 3000 (Invitrogen; Life Technologies Inc.) following the reverse transfection protocol, as described elsewhere ${ }^{28}$. Empty pcDNA3.1(+)-C-6His vector was used as control.

RNA isolation and RT-qPCR analysis of gene expression $\square$

RNA isolation and RT-qPCR for measuring gene expression were performed as described earlier ${ }^{28}$. Predesigned KiCqStart SYBR Green optimized primers from Sigma-Aldrich (KSPQ12012) were used for RT-qPCR.

Protein isolation and Western blotting analysis of protein expression $\square \square$

Protein isolation and Western blotting analysis of protein expression were performed as described earlier ${ }^{28}$. Primary antibodies for puromycin (1:25,000 
dilution; MABE343, Merck), 1:1000 dilution of Esrra (ab16363, Abcam; 07-662, Millipore; and 13826S, CST), Rplp1 (PA5-103540, Invitrogen), Lamp2 (PA1-655, Invitrogen), Ctsd (sc377299, Santa Cruz), Gapdh (2118, CST), cleaved-Casp3 (9661, CST), Map1lc3b-ii/Lc3b (a2775, CST), and Sqstm1/p62 (5114, CST) were used. Horseradish peroxidase-conjugated secondary antibodies recognizing mouse (sc-2954) and rabbit (sc-2955) immunoglobulin Gs (IgGs) were purchased from Santa Cruz Biotechnology. Blots were observed on Image Lab software (BioRad) and densitometric analysis was performed using ImageJ software (NIH, Bethesda, MD, USA) normalized to GAPDH as loading controls.

Immunoprecipitation of puromycin-incorporated proteins $\square$

Protein immunoprecipitation was performed using Dynabeads ${ }^{\mathrm{TM}}$ Protein $\mathrm{G}$ for Immunoprecipitation (Invitrogen) as per manufacturer's protocol. Non-denaturing lysis buffer (Abcam) was used to prepare tissue homogenate. Puromycin (1:5,000 dilution; MABE343, Merck), or $4 \mu \mathrm{g}$ normal rabbit IgG as control (12-370, SigmaAldrich) was used for pull-down assay. Western blot analysis for the detection of pulled down proteins was performed as described above. $\square$

Label-free quantitative proteomic analysis

Label-free quantitative analysis of proteins, recovered from pooled triplicates each group, by LC-MS/MS was performed by NonovogeneAIT (Singapore) as described below.

Protein Quality Test: BSA standard protein solution was prepared according to the instructions of Bradford protein quantitative kit, with gradient concentration ranged from 0 to $0.5 \mathrm{~g} / \mathrm{L}$. BSA standard protein solutions and sample solutions with different 
dilution multiples were added into 96 -well plate to fill up the volume to $20 \mu \mathrm{L}$, respectively. Each gradient was repeated three times. The plate was added $180 \mu \mathrm{L}$ G250 dye solution quickly and placed at room temperature for 5 minutes, the absorbance at $595 \mathrm{~nm}$ was detected. The standard curve was drawn with the absorbance of standard protein solution and the protein concentration of the sample was calculated. $20 \mu \mathrm{g}$ of the protein sample was loaded to $12 \%$ SDS-PAGE gel electrophoresis, wherein the concentrated gel was performed at $80 \mathrm{~V}$ for $20 \mathrm{~min}$, and the separation gel was performed at $120 \mathrm{~V}$ for $90 \mathrm{~min}$. The gel was stained by coomassie brilliant blue R-250 and decolored until the bands were visualized clearly.

Trypsin treatment: $120 \mu \mathrm{g}$ of each protein sample was taken and the volume was made up to $100 \mu \mathrm{L}$ with dissolution buffer, $1.5 \mu \mathrm{g}$ trypsin and $500 \mu \mathrm{L}$ of $100 \mathrm{mM}$ TEAB buffer were added, sample was mixed and digested at $37^{\circ} \mathrm{C}$ for 4 h. Andt hen, $1.5 \mu \mathrm{g}$ trypsin and $\mathrm{CaCl} 2$ were added, sample was digested overnight. Formic acid was mixed with digested sample, adjusted $\mathrm{pH}$ under 3 , and centrifuged at $12000 \mathrm{~g}$ for $5 \mathrm{~min}$ at room temperature. The supernatant was slowly loaded to the C18 desalting column, washed with washing buffer $(0.1 \%$ formic acid, $3 \%$ acetonitrile) 3 times, then eluted by some elution buffer $(0.1 \%$ formic acid, $70 \%$ acetonitrile). The eluents of each sample were combined and lyophilized.

LC-MS/MS Analysis: Mobile phase A (100\% water, $0.1 \%$ formic acid) and B solution ( $80 \%$ acetonitrile, $0.1 \%$ formic acid) were prepared. The lyophilized powder was dissolved in $10 \mu \mathrm{L}$ of solution $\mathrm{A}$, centrifuged at $14,000 \mathrm{~g}$ for $20 \mathrm{~min}$ at $4{ }^{\circ} \mathrm{C}$, and $1 \mu \mathrm{g}$ of the supernatant was injected into a home-made C18 Nano-Trap column (2 $\mathrm{cm} \times 75 \mu \mathrm{m}, 3 \mu \mathrm{m})$. Peptides were separated in a home-made analytical column (15 
$\mathrm{cm} \times 150 \mu \mathrm{m}, 1.9 \mu \mathrm{m})$, using a linear gradient elution. The separated peptides were analyzed by Q Exactive HF-X mass spectrometer (Thermo Fisher), with ion source of Nanospray Flex ${ }^{\mathrm{TM}}$ (ESI, spray voltage of $2.3 \mathrm{kV}$ and ion transport capillary temperature of $320^{\circ} \mathrm{C}$. Full scan range from $\mathrm{m} / \mathrm{z} 350$ to 1500 with resolution of 60000 (at $\mathrm{m} / \mathrm{z} 200$ ), an automatic gain control (AGC) target value was $3 \times 106$ and a maximum ion injection time was $20 \mathrm{~ms}$. The top 40 precursors of the highest abundant in the full scan were selected and fragmented by higher energy collisional dissociation (HCD) and analyzed in MS/MS, where resolution was 15000 (at $\mathrm{m} / \mathrm{z} 200$ ), the automatic gain control (AGC) target value was $1 \times 105$, the maximum ion injection time was 45 ms, a normalized collision energy was set as $27 \%$, an intensity threshold was $2.2 \times 104$, and the dynamic exclusion parameter was $20 \mathrm{~s}$. The raw data of MS detection was named as ".raw".

The identification and quantitation of protein: All resulting spectra were searched against Mus_musculus_uniprot_2019.01.18.fasta (85165 sequences) database by the search engines: Proteome Discoverer 2.2 (PD 2.2, Thermo). The search parameters are set as follows: mass tolerance for precursor ion was $10 \mathrm{ppm}$ and mass tolerance for product ion was $0.02 \mathrm{Da}$. Carbamidomethyl was specified as fixed modifications, Oxidation of methionine (M) was specified as dynamic modification, and acetylation was specified as $\mathrm{N}$-Terminal modification in PD 2.2. A maximum of 2 missed cleavage sites were allowed. In order to improve the quality of analysis results, the software PD 2.2 further filtered the retrieval results: Peptide Spectrum Matches (PSMs) with a credibility of more than $99 \%$ was identified PSMs. The identified protein contains at least 1 unique peptide. The identified PSMs and protein were retained and performed with FDR no more than $1.0 \%$. The protein quantitation results were statistically analyzed by T-test. The proteins whose 
quantitation significantly different between experimental and control groups, $(p<$ 0.05 and $|\log 2 \mathrm{FC}|>=2$ (ratio $>=4$ or ratio $<=0.25$ [fold change, FC]), were defined as differentially expressed proteins (DEP).

The functional analysis of protein and DEP: Gene Ontology (GO) and KEGG (Kyoto Encyclopedia of Genes and Genomes) were used to analyze the protein family and pathway whereas Targeting Protein-Protein Interactions (PPIs) for protein-protein interactions on EnrichR platform (the Ma'ayan Lab, NY, USA) ${ }^{59,60,}$ $61 . \square$

Fluorescence imaging of the cells $\square$

Autophagy flux analysis: ptfLC3 (Addgene plasmid No. 21074) was transfected using lipofectamine 3000 reagent (Invitrogen) in Esrra knockdown or control cells as described in manufacturer's protocol. ptfLC3 was a gift from Tamotsu Yoshimori and described elsewhere ${ }^{62}$. After $48 \square$ h of transfection, cells were serum starved for further $24 \square \mathrm{h}$. The cells were then fixed with $4 \%$ paraformaldehyde for $15 \square \min$ and washed three times with PBS. Slides were washed and wet mounted in VECTASHIELD Antifade Mounting Medium. Fluorescence imaging was performed using LSM710 Carl Zeiss (Carl Zeiss Microscopy GmbH, Oberkochen, Germany) confocal microscope at $40 \times$ magnification.

Acridine orange $(\mathrm{AO})$ staining: Cells were transfected and grown in 24-well plate for $48 \mathrm{~h}$ and serum starved for further $24 \square \mathrm{h}$. Thereafter, cells were incubated with $1 \square \mu \mathrm{g} / \mathrm{ml}$ of $\mathrm{AO}$ (Sigma-Aldrich) in PBS for $30 \square \min$ at $37 \square{ }^{\circ} \mathrm{C}$, and observed under a fluorescence microscope as described elsewhere ${ }^{63}$. $\square$ 
Fresh liver tissue was placed in fixative [2\% paraformaldehyde and $3 \%$ glutaraldehyde in cacodylate buffer $(\mathrm{pH} 7.4)]$ and stored at $4^{\circ} \mathrm{C}$. TEM analysis was performed as described previously ${ }^{28}$. Images were taken using the Olympus EM208S transmission electron microscope (Japan) at $\times 10,000$ magnifications. Mean number of lysosomes per TEM field from untreated control and XCT790-treated mouse liver samples was calculated from a total of 10 random fields per treatment.

Mitochondrial oxygen consumption rate (OCR) measurement by Seahorse extracellular flux analyzer $\square$

Seahorse extracellular flux analyser XFe96 (Agilent) was used for mitostress test, and mitochondrial fatty acid fuel oxidation analysis. Seahorse Wave Desktop software was used for report generation and data analysis while GraphPad PRISM 9 was used for statistical analysis and data presentation. 10,000 AML12 cells were seeded on XFe-96-well culture microplates and the assays were performed as described previously ${ }^{64}$.

Statistical analysis $\square$

Individual culture experiments were performed in triplicate and repeated at least three times independently using matched controls; the data were pooled, and statistical analysis was performed. Results are expressed as mean \pm SD for all in vitro and in vivo experiments. The statistical significance of differences ${ }^{*} \mathrm{P}<0.05$; ${ }^{* *} \mathrm{P}<0.01 ;{ }^{* *} \mathrm{P}<0.001 ;{ }^{* * *} \mathrm{P}<0.0001$ ) were assessed by unpaired one-way ANOVA or two-way ANOVA, followed by Tukey's multiple-comparisons test, wherever applicable. All statistical tests were performed using Prism 9 for Mac OS X (GraphPad Software). 


\section{Abbreviations}

$\mathrm{AO}$, acridine orange $\square$

C29, compound $29 \square \square$

CHX, cycloheximide $\square$

Esrra, Estrogen related receptor alpha $\square$

$\mathrm{KD}$, knockdown $\square$

MCD, methionine choline-deficient $\square$

NAFLD, non-alcoholic fatty liver disease $\square$

$\mathrm{NASH}$, non-alcoholic steatohepatitis $\square$

PA, palmitic acid $\square$

PHHep, primary human hepatocytes $\square$

WT, wild type

\section{Acknowledgements}

This research was funded by the Ministry of Health $(\mathrm{MOH})$ and National Medical

Research Council (NMRC), Singapore, grant numbers NMRC/OFYIRG/0002/2016 and MOH-000319 (MOH-OFIRG19may-0002) to B.K.S.; NMRC/OFYIRG/077/2018 to M.T.; and CSAl19may-0002 to P.M.Y. 


\section{Figure legends $\square$}

Fig. 1 Rate of protein translation was temporally regulated in NASH and starvation, and regulated by Esrra-Rplp1 axis. (A and $C$ ) Representative Western blots of puromycin-incorporated proteins in serum starved primary human hepatocytes (PHHep; $n=3$ per group), and AML12 cells ( $n=3$ per group). (B and D) Plots represent relative density of corresponding Western blot normalized to Gapdh. (E) Graph represents the percent of total proteins upregulated $>50 \%$ of expression analyzed from a label-free quantification by mass spectrometry. (F) Venn analysis for common and exclusive pathways regulated under 48 (blue) and $72 \mathrm{~h}$ (orange) serum starvation. $(G$ and $H)$ Heat maps representing the differential expression of proteins regulating ribosomes $(\mathrm{G})$ under and lysosomes $(\mathrm{H})$ in 48 and $72 \mathrm{~h}$ serum starved AML12 cells. (I) Puromycin-incorporated proteins immunoprecipitation for newly synthesized Rplp1, Lamp2, and Ctsd using Western blotting. Figure is showing a representative Western blot $n=3$. (J) Transcription factor analysis for $>50 \%$ expressed proteins in $72 \mathrm{~h}$ serum starved AML12 cells compared to $48 \mathrm{~h}$ using Targeting Protein-Protein Interactions (PPIs) on EnrichR platform (the Ma'ayan Lab, NY, USA). (K) Representative Western blots of AML12 cells treated control siRNA or Esrra siRNA for 72 h. (L) Plot represents relative density of Western blots normalized to Gapdh. (M) Microscopic image showing acridine orange staining of AML12 cells treated with control siRNA or Esrra siRNA with or without $24 \mathrm{~h}$ serum starvation. Images are representative of three fields per group and three independent experiments. Scale bars, $200 \mu \mathrm{m}(\mathrm{N})$ Plot shows relative fluorescence of $\mathrm{K}$ that was measured using ImageJ $(\mathrm{NIH}, \mathrm{USA})$. 
$\mathrm{n}=5$ /groups. (O) Representative Western blots of AML12 cells treated with control, Esrra or Rplp1 siRNA for 72 h. (P and Q) Plots represent relative density of Western blots normalized to Gapdh. (R) Representative Western blots of AML12 cells treated with empty plasmid, Esrra or Rplp1 expressing plasmid for $72 \mathrm{~h}$. (S and T) Plots represents relative density of Western blots normalized to Gapdh.

Levels of significance: ${ }^{*} \mathrm{P}<0.05 ;{ }^{* *} \mathrm{P}<0.01$; ${ }^{* \star} \mathrm{P}<0.001$; ${ }^{* \star *} \mathrm{P}<0.0001$; (One way or Two way ANOVA followed by Tukey's test).

Fig. 2. Temporally regulated autophagy was associated with Rplp1-lysosome axis and newly synthesized autophagy proteins under starvation. (A) Representative Western blots of serum starved AML12 cells for indicated time points ( $n=3$ per group). ( $B$ and $C$ ) Plots represent relative density of corresponding Western blot normalized to Gapdh showing temporal changes in the proteins. (D) Immunoprecipitation analysis for puromycin-incorporated proteins for newly synthesized Map1lc3b, Sqstm1, Atg5, Med1, and Atg7 using Western blotting. Figure is showing a representative Western blot $n=3$. (E) Microscopic image showing RFP-GFP-LC3 expression in AML12 cells treated with control siRNA or Esrra siRNA with or without $24 \mathrm{~h}$ serum starvation. Images are representative of three fields per group and three independent experiments. Scale bars, $5 \mu \mathrm{m}$. (F) Representative Western blots of AML12 cells treated control siRNA or Esrra siRNA with or without bafilimycin A1 (Baf) for $4 \mathrm{~h}$. (G) Plot represents relative density of Western blots normalized to Gapdh. $(\mathrm{H})$ Representative Western blots of AML12 cells treated empty plasmid or Esrra expressing plasmid with or 
without bafilimycin A1 (Baf) for $4 \mathrm{~h}$. (I) Plot represents relative density of Western blots normalized to Gapdh.

Levels of significance: ${ }^{*} \mathrm{P}<0.05$; (One way or Two way ANOVA followed by Tukey's test). $\square$

\section{Fig. 3. Esrra regulated temporal changes in Rplp1-lysosome axis and} autophagy in mice livers. (A) Representative Western blots of fed and $24 \mathrm{~h}$ starved livers from WT and Esrra KO mice ( $n=5$ per group). (B) Plots represent relative density of corresponding Western blots normalized to Gapdh. (C) Representative Western blots of livers from liver-specific overexpressed Esrra (Alb-mEsrra) or control ( $A / b$-null) mice ( $\mathrm{n}=5$ per group). (D) Plots represent relative density of corresponding Western blots normalized to Gapdh. (E) Representative Western blots of starved mice livers at indicated time points treated with vehicle or Esrra inhibitor C29 ( $n=5$ per group). (F-J) Plots represent relative density of corresponding Western blots normalized to Gapdh.

Levels of significance: ${ }^{*} \mathrm{P}<0.05 ;{ }^{* *} \mathrm{P}<0.01$; ${ }^{* *} \mathrm{P}<0.001$; ${ }^{* * *} \mathrm{P}<0.0001$; (One way or Two way ANOVA followed by Tukey's test).

\section{Fig. 4. Defective Esrra-Rplp1 pathway leading to decreased translation rate} and lysosome-autophagy in NASH livers. (A) Representative Western blots of human livers collected from steatosis and NASH patients compared to control subjects ( $n=5$ per group). (B) Plots represent relative density of corresponding Western blots normalized to Gapdh. (C) Representative micrograph of H\&E staining in liver tissues collected from mice fed WDF for 8 
and 16 weeks to generate steatosis and NASH and compared to controls fed normal chow diet (NCD) ( $=5$ per group). Scale bars are $100 \mu \mathrm{M}$. (D) Representative Western blots of in livers collected from mice fed WDF for 8 and 16 weeks to generate steatosis and NASH and compared to controls fed normal chow diet (NCD) ( $n=5$ per group). (E) Plots represent relative density of corresponding Western blots normalized to Gapdh. (F) Representative micrograph of $\mathrm{H} \& \mathrm{E}$ staining in liver tissues collected from mice fed NCD or MCD diet for 3 and 6 weeks ( $n=5$ per group). Scale bars are $100 \mu M$. (G) Representative Western blots of livers from mice fed NCD or MCD diet for 3 and 6 weeks ( $\mathrm{n}=5$ per group). ( $\mathrm{H}$ and $\mathrm{I}$ ) Plots represent relative density of corresponding Western blots normalized to Gapdh. $\square(\mathrm{J})$ Representative Western blots of $24 \mathrm{~h}$ PA (0.5 mM) treated PHHep ( $\mathrm{n}=3-5$ per group). (K and L) Plots represent relative density of corresponding Western blots normalized to Gapdh. (M) Representative Western blots of 24 h PA $(0.5 \mathrm{mM})$ treated AML12 cells ( $n=3-5$ per group). ( $N$ and O) Plots represent relative density of corresponding Western blots normalized to Gapdh.

Levels of significance: ${ }^{*} \mathrm{P}<0.05$; ${ }^{* *} \mathrm{P}<0.01$; ${ }^{* *} \mathrm{P}<0.001$; ${ }^{* * *} \mathrm{P}<0.0001$; (One way or Two way ANOVA followed by Tukey's test).

Fig. 5. Hepatic Esrra overexpression restored Rplp1-lysosome axis and improved lipotoxicity NASH-related inflammation and fibrosis gene expressions in vitro and NASH in vivo. (A) Representative Western blots of 24 h PA (0.5 mM) treated AML12 cells overexpressed with Esrra or Rplp1 or empty vector. (B and C) Plots represent relative density of corresponding Western blots normalized to Gapdh. (D) Representative Western blots of 
livers from null and hepatic-Esrra overexpressed mice fed NCD or WDF for 8 weeks ( $n=5$ per group). Esrra Western blots were low exposed (L. Exp) and high exposed (H. Exp) for accurate densitometric measurements of Esrra overexpression and basal Esrra expression. (E) Plots represent relative density of corresponding Western blots normalized to Gapdh. (F-I) Measurements of liver index, fasting serum $\beta-\mathrm{HB}$, liver TG, and fasting blood glucose measurements in null and hepatic-Esrra overexpressed mice fed NCD or WDF for 16 weeks. $\square(\mathrm{J})$ RT-qPCR analysis of relative gene expression in livers from null and hepatic-Esrra overexpressed mice fed NCD or WDF for 8 weeks ( $n=5$ per group). Gene expression was normalized to Gapdh. (L) Representative micrograph of H\&E staining in liver tissues collected from null and hepatic-Esrra overexpressed mice fed NCD or WDF for 8 weeks ( $n=5$ per group). Scale bars are $100 \mu M$.

Levels of significance: ${ }^{*} \mathrm{P}<0.05$; ${ }^{* \star} \mathrm{P}<0.01$; ${ }^{* \star} \mathrm{P}<0.001$; ${ }^{* \star *} \mathrm{P}<0.0001$; (One way or Two way ANOVA followed by Tukey's test).

\section{Fig. 6. Esrra-Rplp1 axis was required for improved translation and lysosome-} autophagy during fasting in NASH liver. (A) Scheme shows alternate day fasting regime in NASH mice: WDF fed mice for 16 weeks ( $n=5$ per group) fasted and fed alternate days and injected vehicle or C29 during fasting days only. After five Fast-Fed cycles, blood and liver tissue were harvested for analysis. Untreated mice fed NCD or WDF for 16 weeks were used for comparison ( $n=4-5$ per group). (B) Representative Western blots of livers from puromycin injected WDF fed mice ( $\mathrm{n}=5$ per group) fasted and fed alternate days for 5 consecutive cycles and injected vehicle or C29 during fasting days 
(as shown in Panel A). Untreated mice fed NCD or WDF for 16 weeks were used for comparison ( $n=4-5$ per group). (C) Plot represents relative density of Western blot normalized to Gapdh. (D) Representative Western blots of livers as described in panel A. (E) Plots represent relative density of Western blots normalized to Gapdh. ( $F$ and $G$ ) Serum $\beta$-HB measurement and liver index measurements in the livers from the mice described in panel $A .(H) R T-q P C R$ analysis of relative gene expression in the livers from the mice described in panel A. Gene expression was normalized to Gapdh $(\mathrm{n}=4-5)$. (I) Representative micrograph of H\&E staining in liver tissues collected from mice as described in panel $A$ ( $n=5$ per group). Scale bars are $100 \mu M$.

Levels of significance: ${ }^{*} \mathrm{P}<0.05 ;{ }^{* *} \mathrm{P}<0.01$; ${ }^{* \star} \mathrm{P}<0.001$; ${ }^{* \star *} \mathrm{P}<0.0001$; (One way or Two way ANOVA followed by Tukey's test). 


\section{References}

1. Eslam M, Sanyal AJ, George J, International Consensus P. MAFLD: A ConsensusDriven Proposed Nomenclature for Metabolic Associated Fatty Liver Disease. Gastroenterology 158, 1999-2014 e1991 (2020).

2. Lim S, Kim JW, Targher G. Links between metabolic syndrome and metabolic dysfunction-associated fatty liver disease. Trends Endocrinol Metab, (2021).

3. Loomba R, Friedman SL, Shulman GI. Mechanisms and disease consequences of nonalcoholic fatty liver disease. Cell 184, 2537-2564 (2021).

4. Younossi ZM, et al. Nonalcoholic Steatohepatitis Is the Most Rapidly Increasing Indication for Liver Transplantation in the United States. Clin Gastroenterol Hepatol 19, 580-589 e585 (2021).

5. Drinda S, et al. Effects of Periodic Fasting on Fatty Liver Index-A Prospective Observational Study. Nutrients 11, (2019).

6. Hatchwell L, et al. Multi-omics Analysis of the Intermittent Fasting Response in Mice Identifies an Unexpected Role for HNF4alpha. Cell Rep 30, 3566-3582 e3564 (2020).

7. Johari MI, et al. A Randomised Controlled Trial on the Effectiveness and Adherence of Modified Alternate-day Calorie Restriction in Improving Activity of NonAlcoholic Fatty Liver Disease. Sci Rep 9, 11232 (2019).

8. Viveiros K. The Role of Life Style Modifications in Comprehensive Non-Alcoholic Fatty Liver Disease Treatment. Clin Liver Dis (Hoboken) 17, 11-14 (2021).

9. Brocker $\mathrm{CN}$, et al. Extrahepatic PPARalpha modulates fatty acid oxidation and attenuates fasting-induced hepatosteatosis in mice. J Lipid Res 59, 2140-2152 (2018).

10. Li G, et al. Metabolic adaptation to intermittent fasting is independent of peroxisome proliferator-activated receptor alpha. Mol Metab 7, 80-89 (2018).

11. Rasineni K, et al. Contrasting Effects of Fasting on Liver-Adipose Axis in AlcoholAssociated and Non-alcoholic Fatty Liver. Front Physiol 12, 625352 (2021).

12. Jaud M, et al. Translational Regulations in Response to Endoplasmic Reticulum Stress in Cancers. Cells 9, (2020).

13. Yin Z, et al. Psp2, a novel regulator of autophagy that promotes autophagy-related protein translation. Cell Res 29, 994-1008 (2019).

14. Fonseca BD, Smith EM, Yelle N, Alain T, Bushell M, Pause A. The ever-evolving role of mTOR in translation. Semin Cell Dev Biol 36, 102-112 (2014).

15. Tahmasebi S, Khoutorsky A, Mathews MB, Sonenberg N. Translation deregulation in human disease. Nat Rev Mol Cell Biol 19, 791-807 (2018). 
16. Hwang J, Qi L. Quality Control in the Endoplasmic Reticulum: Crosstalk between ERAD and UPR pathways. Trends Biochem Sci 43, 593-605 (2018).

17. Koo JH, Han CY. Signaling Nodes Associated with Endoplasmic Reticulum Stress during NAFLD Progression. Biomolecules 11, (2021).

18. Rashid HO, Yadav RK, Kim HR, Chae HJ. ER stress: Autophagy induction, inhibition and selection. Autophagy 11, 1956-1977 (2015).

19. Senft D, Ronai ZA. UPR, autophagy, and mitochondria crosstalk underlies the ER stress response. Trends Biochem Sci 40, 141-148 (2015).

20. Sinha RA, Singh BK, Yen PM. Reciprocal Crosstalk Between Autophagic and Endocrine Signaling in Metabolic Homeostasis. Endocr Rev 38, 69-102 (2017).

21. Cadwell K. Crosstalk between autophagy and inflammatory signalling pathways: balancing defence and homeostasis. Nat Rev Immunol 16, 661-675 (2016).

22. Goldsmith J, et al. Ribosome profiling reveals a functional role for autophagy in mRNA translational control. Commun Biol 3, 388 (2020).

23. Allaire M, Rautou PE, Codogno P, Lotersztajn S. Autophagy in liver diseases: Time for translation? J Hepatol 70, 985-998 (2019).

24. Engin A. Non-Alcoholic Fatty Liver Disease. Adv Exp Med Biol 960, 443-467 (2017).

25. Park HS, et al. TXNIP/VDUP1 attenuates steatohepatitis via autophagy and fatty acid oxidation. Autophagy, 1-16 (2020).

26. Aviner R. The science of puromycin: From studies of ribosome function to applications in biotechnology. Comput Struct Biotechnol J 18, 1074-1083 (2020).

27. Goodman CA, Hornberger TA. Measuring protein synthesis with SUnSET: a valid alternative to traditional techniques? Exerc Sport Sci Rev 41, 107-115 (2013).

28. Singh BK, et al. Thyroid hormone receptor and ERRalpha coordinately regulate mitochondrial fission, mitophagy, biogenesis, and function. Sci Signal 11, (2018).

29. Feng Y, He D, Yao Z, Klionsky DJ. The machinery of macroautophagy. Cell Res 24, 24-41 (2014).

30. Manning BD. Adaptation to starvation: translating a matter of life or death. Cancer Cell 23, 713-715 (2013).

31. Zhou J, et al. MED1 mediator subunit is a key regulator of hepatic autophagy and lipid metabolism. Autophagy, 1-19 (2021).

32. Dufour CR, et al. Genomic convergence among ERRalpha, PROX1, and BMAL1 in the control of metabolic clock outputs. PLoS Genet 7, e1002143 (2011). 
33. Tripathi M, Yen PM, Singh BK. Estrogen-Related Receptor Alpha: An UnderAppreciated Potential Target for the Treatment of Metabolic Diseases. Int J Mol Sci 21, (2020).

34. Laganiere J, Tremblay GB, Dufour CR, Giroux S, Rousseau F, Giguere V. A polymorphic autoregulatory hormone response element in the human estrogen-related receptor alpha (ERRalpha) promoter dictates peroxisome proliferator-activated receptor gamma coactivator-1 alpha control of ERRalpha expression. J Biol Chem 279, 18504-18510 (2004).

35. Widjaja AA, et al. Inhibiting Interleukin 11 Signaling Reduces Hepatocyte Death and Liver Fibrosis, Inflammation, and Steatosis in Mouse Models of Nonalcoholic Steatohepatitis. Gastroenterology 157, 777-792 e714 (2019).

36. Gao Y, Wang Y, Liu H, Liu Z, Zhao J. Mitochondrial DNA from hepatocytes induces upregulation of interleukin-33 expression of macrophages in nonalcoholic steatohepatitis. Dig Liver Dis 52, 637-643 (2020).

37. Kanuri G, Bergheim I. In vitro and in vivo models of non-alcoholic fatty liver disease (NAFLD). Int J Mol Sci 14, 11963-11980 (2013).

38. Dong J, et al. Hepatocyte-specific IL11 cis-signaling drives lipotoxicity and underlies the transition from NAFLD to NASH. Nat Commun 12, 66 (2021).

39. Geng Y, Faber KN, de Meijer VE, Blokzijl H, Moshage H. How does hepatic lipid accumulation lead to lipotoxicity in non-alcoholic fatty liver disease? Hepatol Int 15, 21-35 (2021).

40. Lynch C, et al. Identification of Estrogen-Related Receptor alpha Agonists in the Tox21 Compound Library. Endocrinology 159, 744-753 (2018).

41. Teng CT, et al. Development of Novel Cell Lines for High-Throughput Screening to Detect Estrogen-Related Receptor Alpha Modulators. SLAS Discov 22, 720-731 (2017).

42. de Cabo R, Mattson MP. Effects of Intermittent Fasting on Health, Aging, and Disease. N Engl J Med 381, 2541-2551 (2019).

43. Yuan HX, Xiong Y, Guan KL. Nutrient sensing, metabolism, and cell growth control. Mol Cell 49, 379-387 (2013).

44. Salazar G, et al. SQSTM1/p62 and PPARGC1A/PGC-1alpha at the interface of autophagy and vascular senescence. Autophagy 16, 1092-1110 (2020).

45. Holmer M, et al. Treatment of NAFLD with intermittent calorie restriction or lowcarb high-fat diet - a randomised controlled trial. JHEP Rep 3, 100256 (2021). 
46. Vilar-Gomez E, et al. Weight Loss Through Lifestyle Modification Significantly Reduces Features of Nonalcoholic Steatohepatitis. Gastroenterology 149, 367-378 e365; quiz e314-365 (2015).

47. Kim SY, et al. ESRRA (estrogen-related receptor alpha) is a key coordinator of transcriptional and post-translational activation of autophagy to promote innate host defense. Autophagy 14, 152-168 (2018).

48. Casaburi I, et al. Estrogen related receptor alpha (ERRalpha) a promising target for the therapy of adrenocortical carcinoma (ACC). Oncotarget 6, 25135-25148 (2015).

49. Kim S, et al. Clinicopathological Profiling of LC3B, an Autophagy Marker, and ESRRA (Estrogen-related Receptor-alpha) in Muscle-invasive Bladder Cancer. Anticancer Res 38, 2429-2437 (2018).

50. Suresh SN, et al. Modulation of Autophagy by a Small Molecule Inverse Agonist of ERRalpha Is Neuroprotective. Front Mol Neurosci 11, 109 (2018).

51. Machado MV, et al. Mouse models of diet-induced nonalcoholic steatohepatitis reproduce the heterogeneity of the human disease. PLoS One 10, e0127991 (2015).

52. Ravi V, Jain A, Ahamed F, Fathma N, Desingu PA, Sundaresan NR. Systematic evaluation of the adaptability of the non-radioactive SUnSET assay to measure cardiac protein synthesis. Sci Rep 8, 4587 (2018).

53. Schmidt EK, Clavarino G, Ceppi M, Pierre P. SUnSET, a nonradioactive method to monitor protein synthesis. Nat Methods 6, 275-277 (2009).

54. Chaveroux C, et al. Molecular and genetic crosstalks between mTOR and ERRalpha are key determinants of rapamycin-induced nonalcoholic fatty liver. Cell Metab 17, 586-598 (2013).

55. Sailland J, et al. Estrogen-related receptor alpha decreases RHOA stability to induce orientated cell migration. Proc Natl Acad Sci U S A 111, 15108-15113 (2014).

56. Bruinstroop E, et al. Early induction of hepatic deiodinase type 1 inhibits hepatosteatosis during NAFLD progression. Mol Metab, 101266 (2021).

57. Tripathi M, Yen PM, Singh BK. Protocol to Generate Senescent Cells from the Mouse Hepatic Cell Line AML12 to Study Hepatic Aging. STAR Protoc 1, 100064 (2020).

58. Widjaja AA, et al. Redefining IL11 as a regeneration-limiting hepatotoxin and therapeutic target in acetaminophen-induced liver injury. Sci Transl Med 13, (2021).

59. Chen EY, et al. Enrichr: interactive and collaborative HTML5 gene list enrichment analysis tool. BMC Bioinformatics 14, 128 (2013).

60. Kuleshov MV, et al. Enrichr: a comprehensive gene set enrichment analysis web server 2016 update. Nucleic Acids Res 44, W90-97 (2016). 
61. Xie Z, et al. Gene Set Knowledge Discovery with Enrichr. Curr Protoc 1, e90 (2021).

62. Kimura N, et al. Expression of autophagy-associated genes in skeletal muscle: an experimental model of chloroquine-induced myopathy. Pathobiology 74, 169-176 (2007).

63. Tripathi M, et al. Hyperhomocysteinemia causes ER stress and impaired autophagy that is reversed by Vitamin B supplementation. Cell Death Dis 7, e2513 (2016).

64. Singh BK, Tripathi M, Sandireddy R, Tikno K, Zhou J, Yen PM. Decreased autophagy and fuel switching occur in a senescent hepatic cell model system. Aging (Albany NY) 12, 13958-13978 (2020). 
(which was not certified by peer review) is the author/funder, who has granted bioRxiv a license to display the preprint in perpetuity. It is $m$ available ruhgè lace $\&_{B Y} 4.0$ International license.

A Serum starvation

$$
\begin{array}{lllll}
0 & 6 & 24 & 48 & 72 \mathrm{~h}
\end{array}
$$

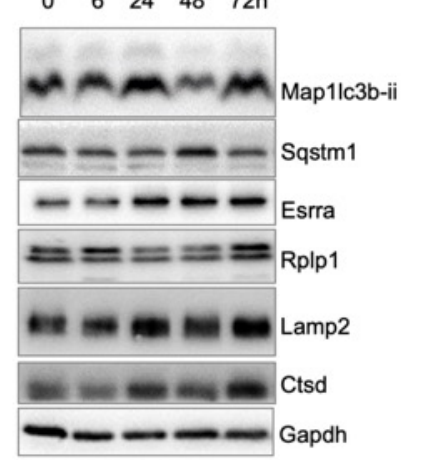

E
B

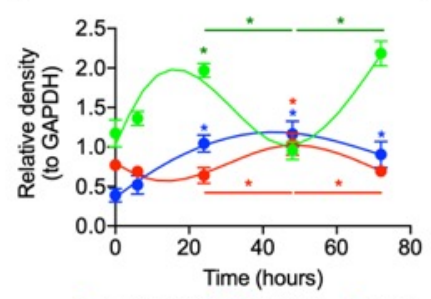

- Map1lc3b-ii * Sqstm1 * Esrra
Overlay F

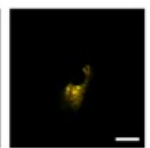

Control siRNA -Serum

Esrra SiRNA

Esrra SiRNA -Serum
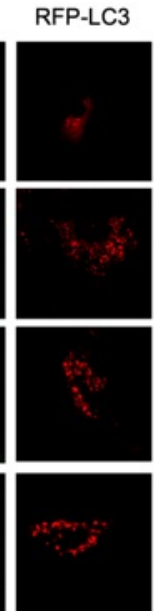

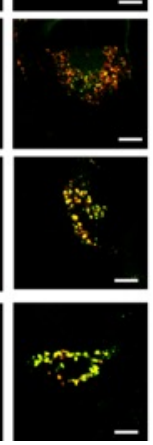

C

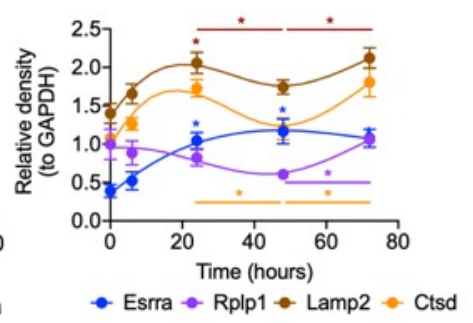

D

Serum starvation
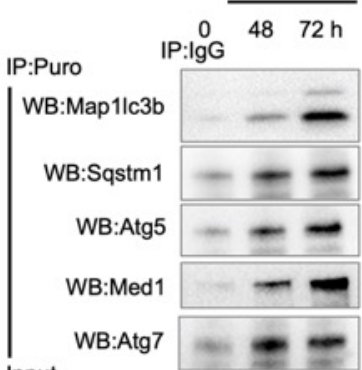

Input

WB:Map1lc3b

WB:Sqstm1

WB:Atg5

WB:Med1
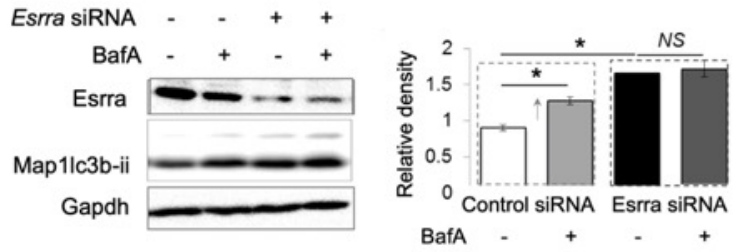

G

H
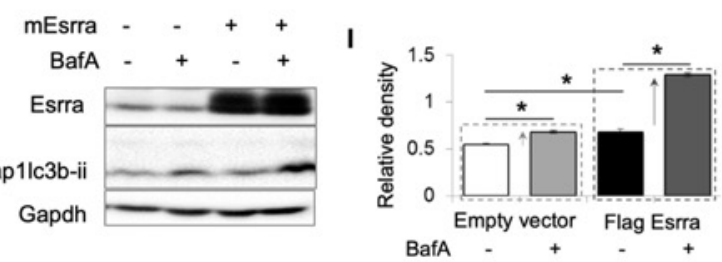

WB:Atg7 
(which was not certified by peer review) is the author/funder, who has granted bioRxiv a license to display the preprint in perpetuity. It is $m$ available angè lac ${ }_{B}$ BY 4.0 International license.

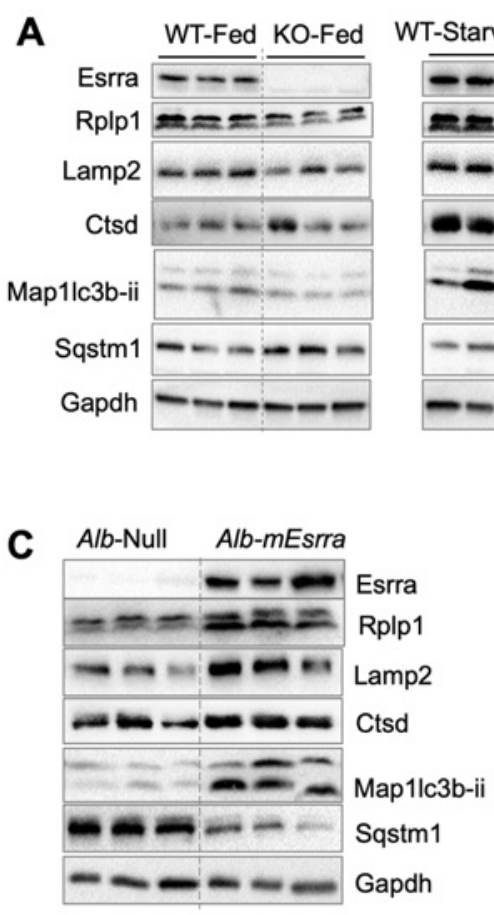

E

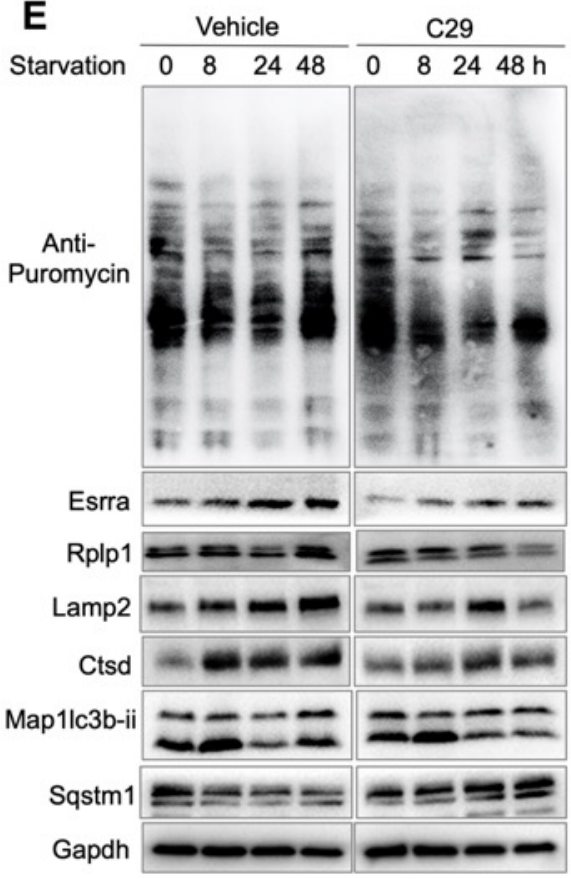

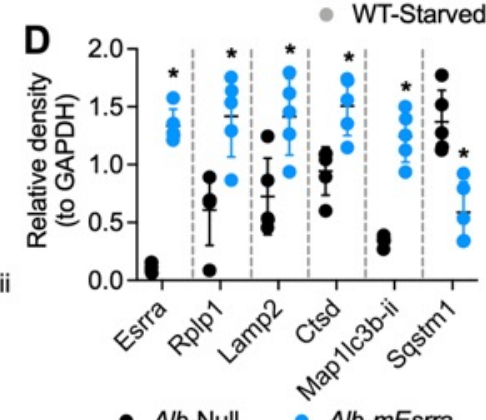

- Alb-Null Alb-mEsrra

$\mathbf{F}$

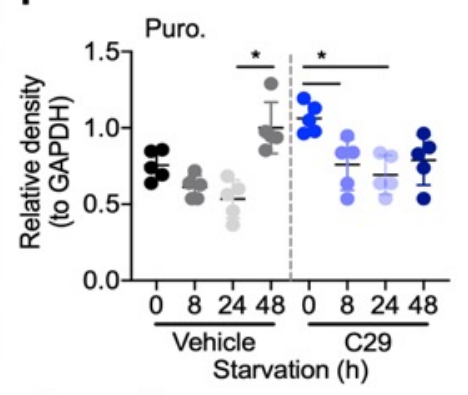

G Esrra

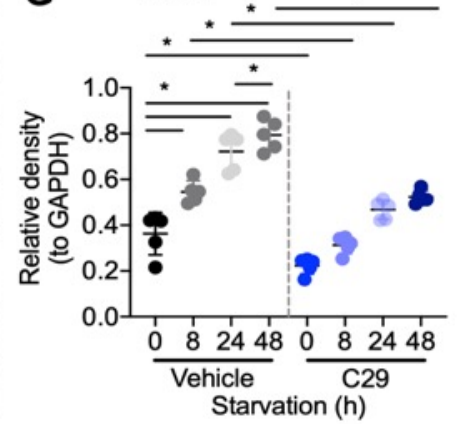

H

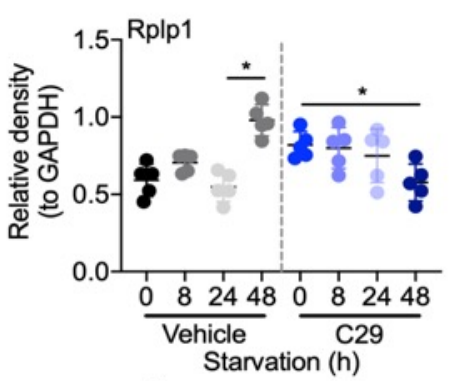

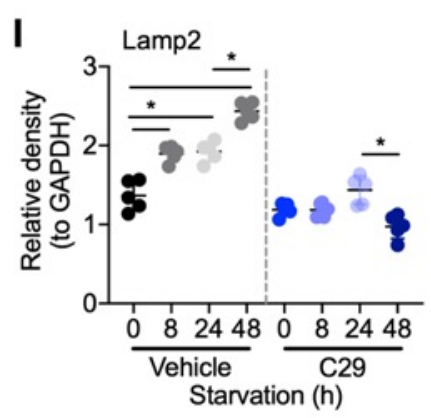
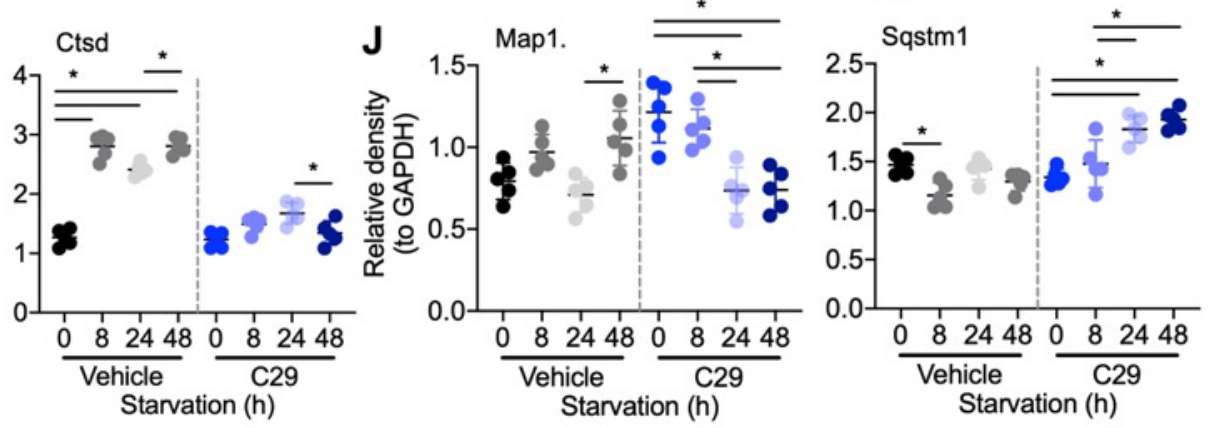
(which was not certified by peer review) is the author/funder, who has granted bioRxiv a license to display the preprint in perpetuity. It is $m$ available angèt lace BY $_{4} .0$ International license.

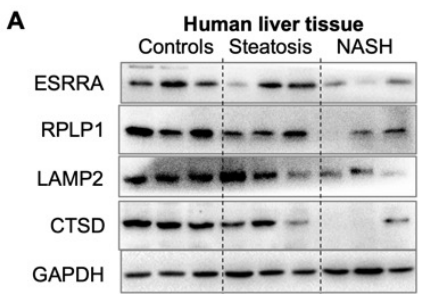

D

\section{Controls Steatosis NASH} $\begin{array}{ccc}\begin{array}{c}\text { Controls } \\ \text { (NCD) }\end{array} & \begin{array}{c}\text { Steatosis } \\ \text { (WDF 8w) }\end{array} & \text { NASH } \\ & \text { (WDF 16 w) }\end{array}$
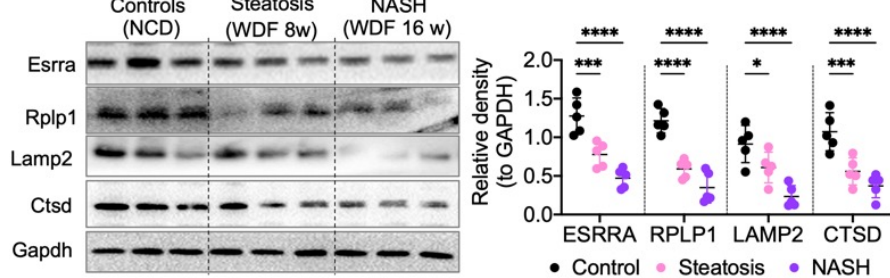

C

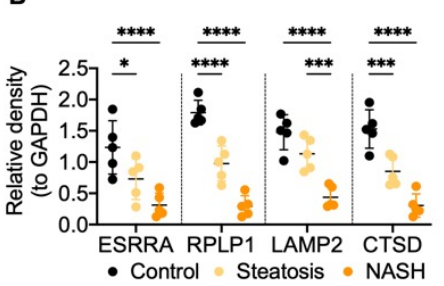

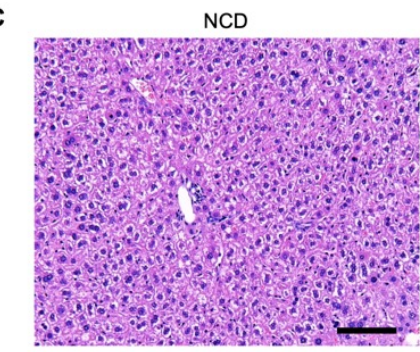

WDF 8w

WDF $16 \mathrm{w}$

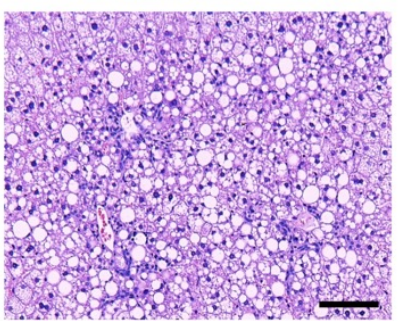

MCD 3w

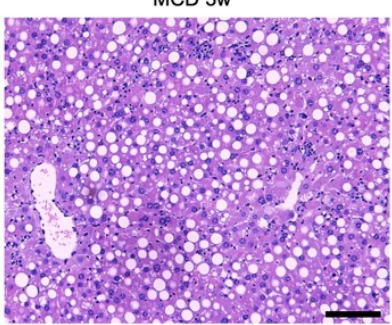

MCD 6w

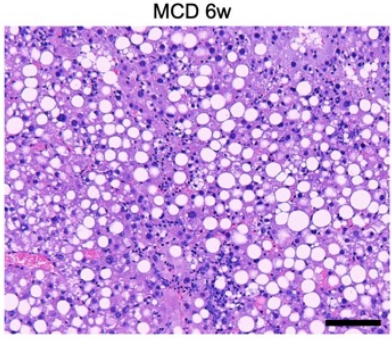

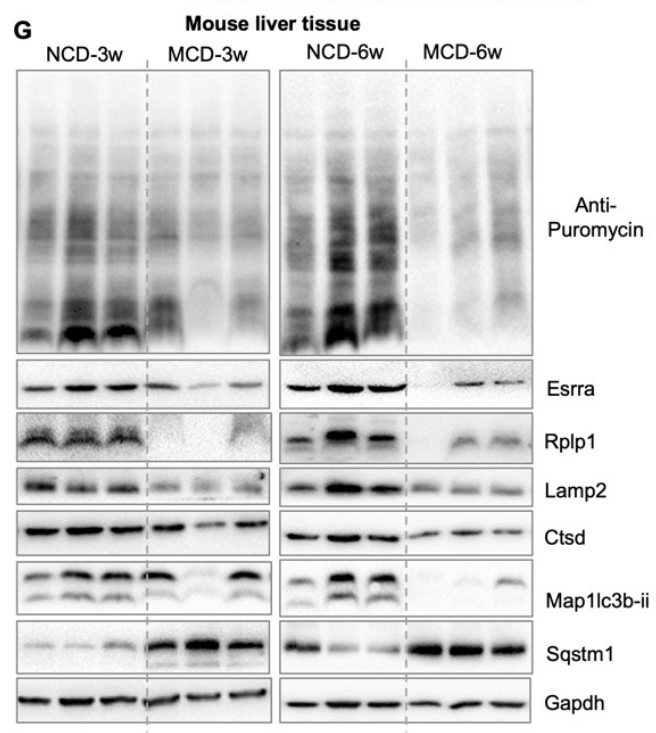

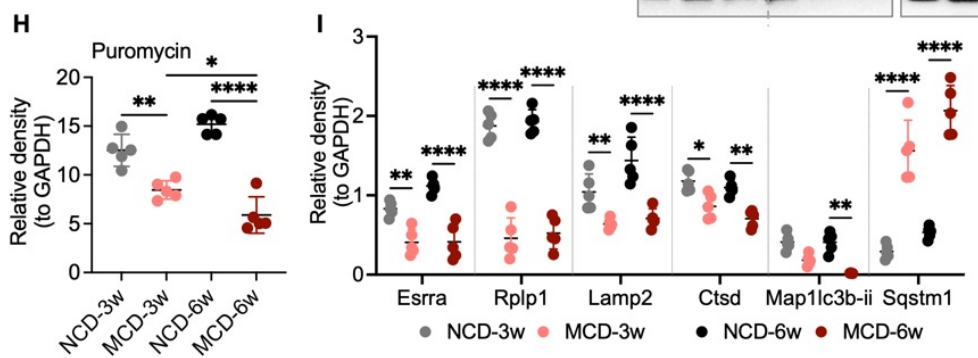

J $\mathrm{PHHep}$

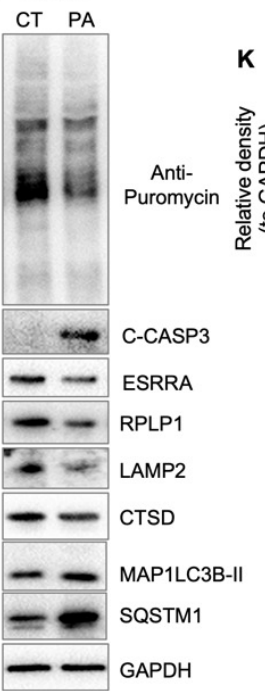

M AML12 cells

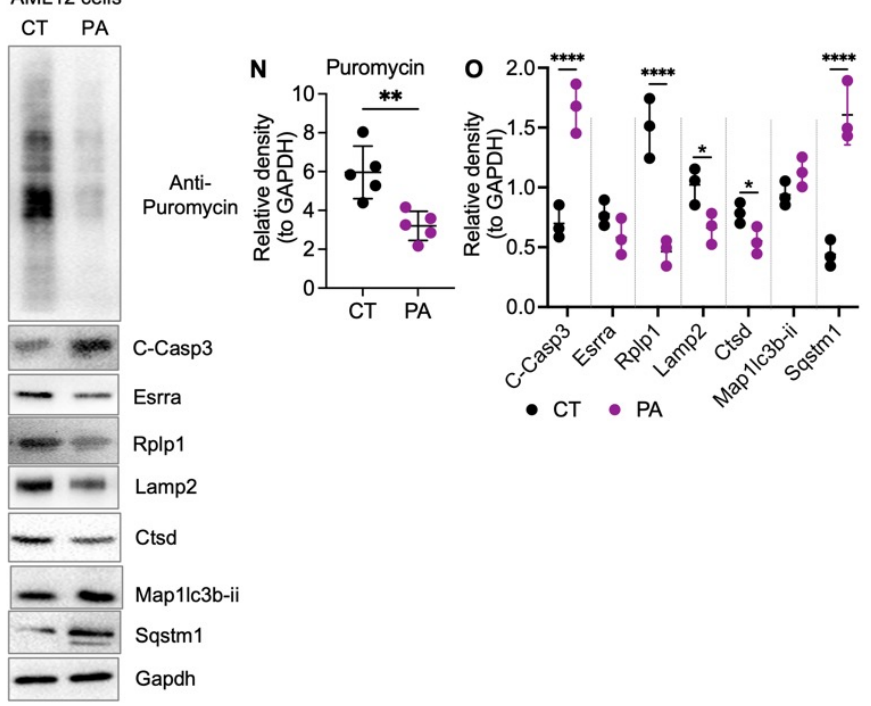


(which was not certified by peer review) is the author/funder, who has granted bioRxiv a license to display the preprint in perpetuity. It is $m$

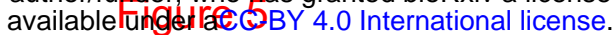

A
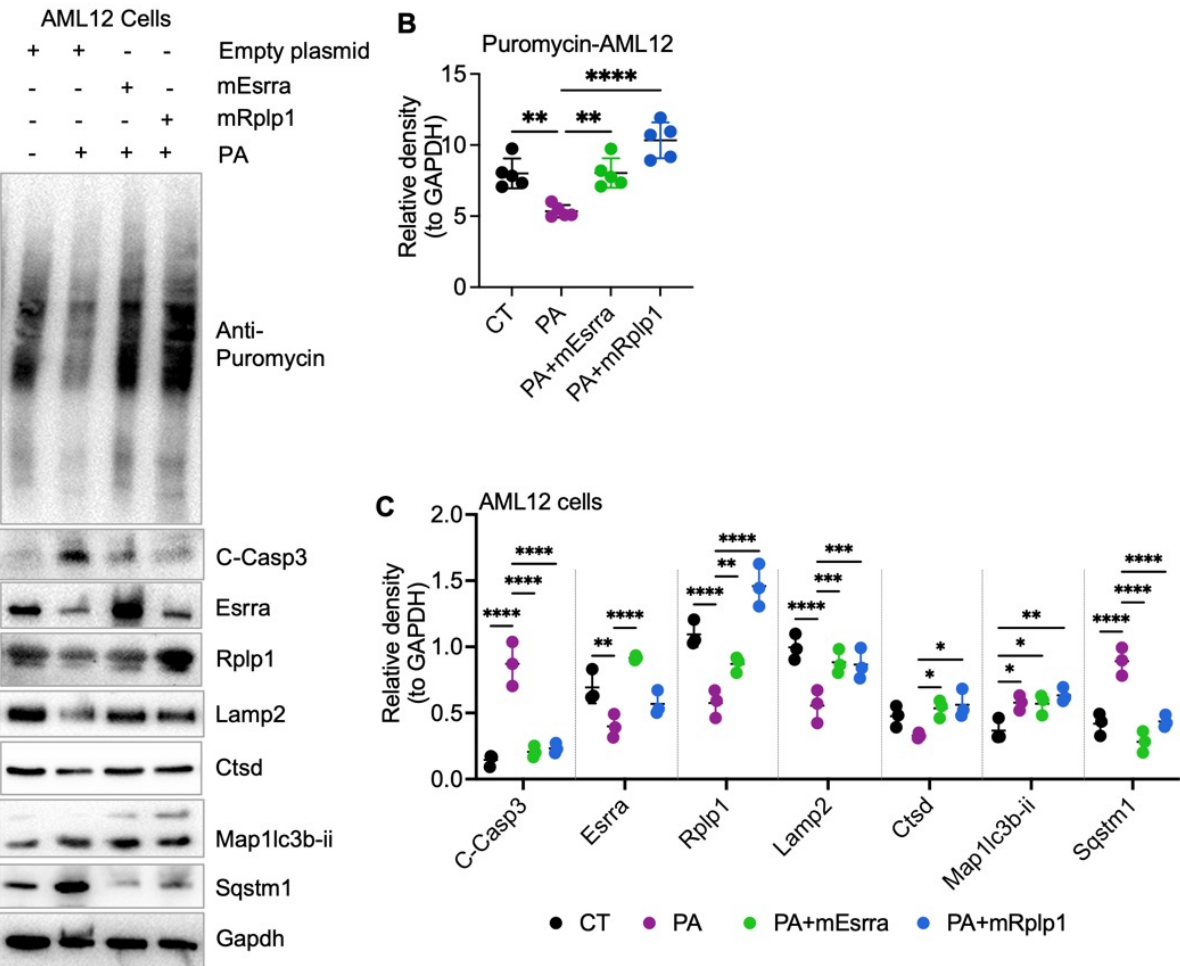

D Null-NCD, Null-WDF, Alb-mEsrra-WDF
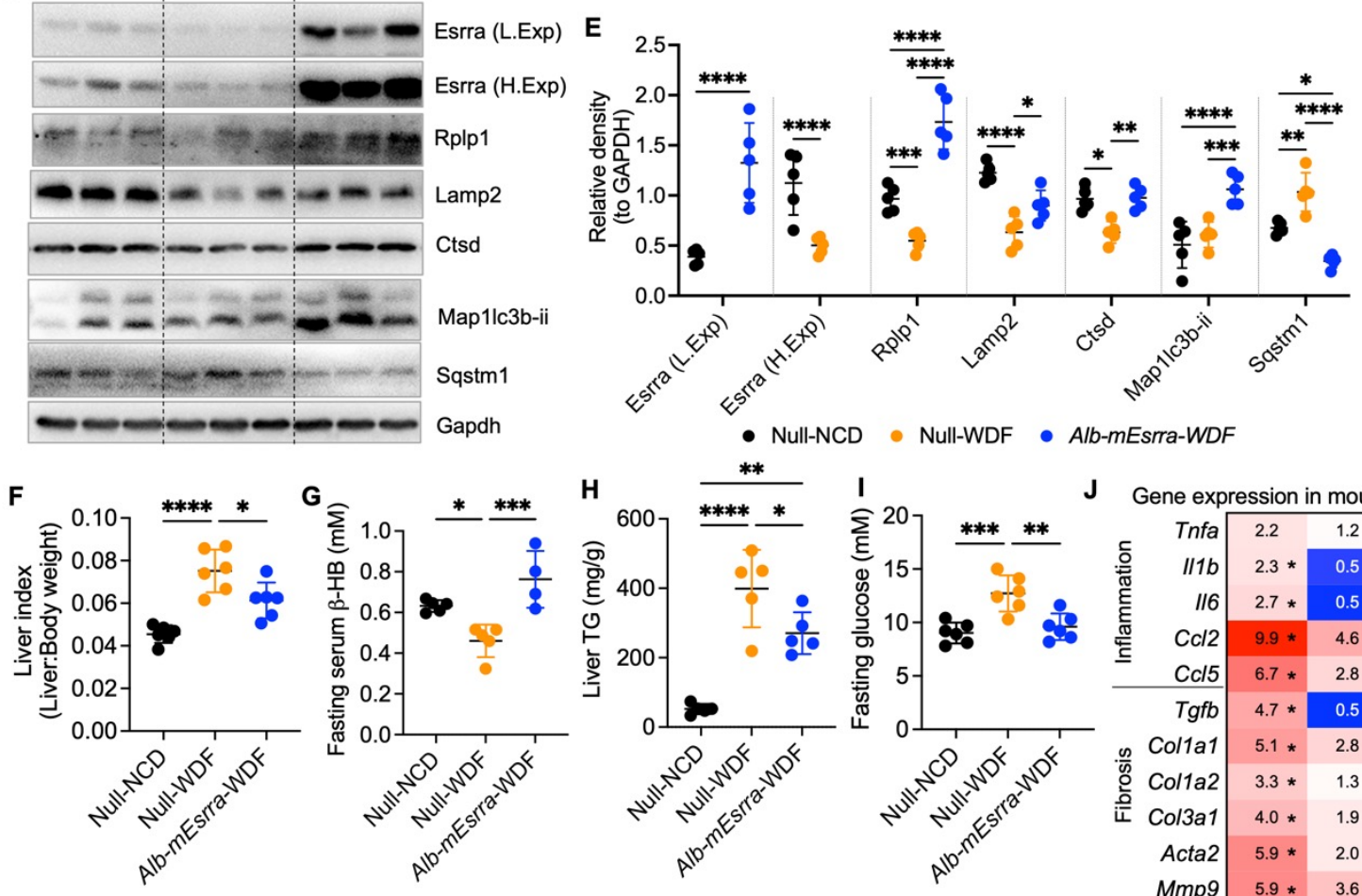

J Gene expression in mouse liver

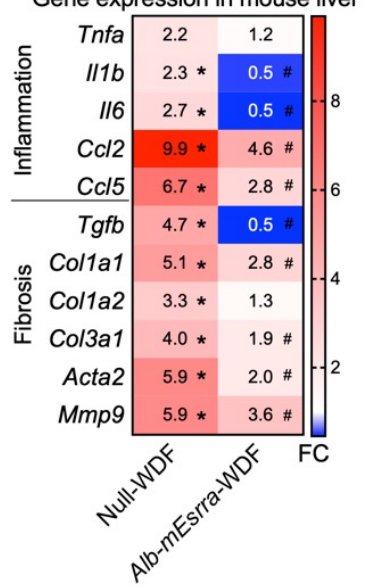

K
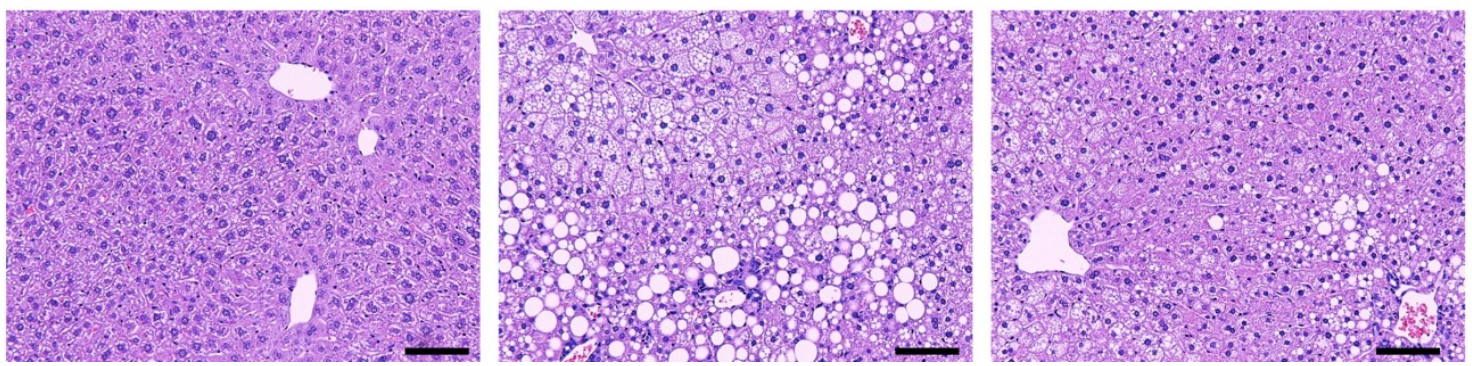
(which was not certified by peer review) is the author/funder, who has granted bioRxiv a license to display the preprint in perpetuity. It is $m$ available angèr fae 6 BY 4.0 International license.

A

16 weeks fed

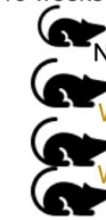

Fed ad libitum

F

Fed baseline

measurement

Fast-Fed Fast-Fed Fast-Fed

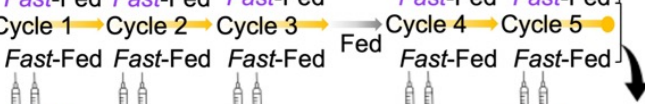
C29 or vehicle injected twice i.p. on Fast days $=$ Euthanize

D Mice liver tissue

NCD W WDF

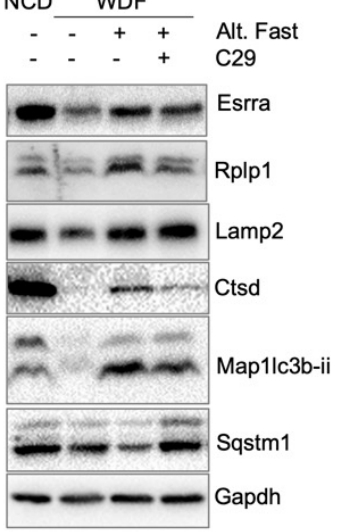

G
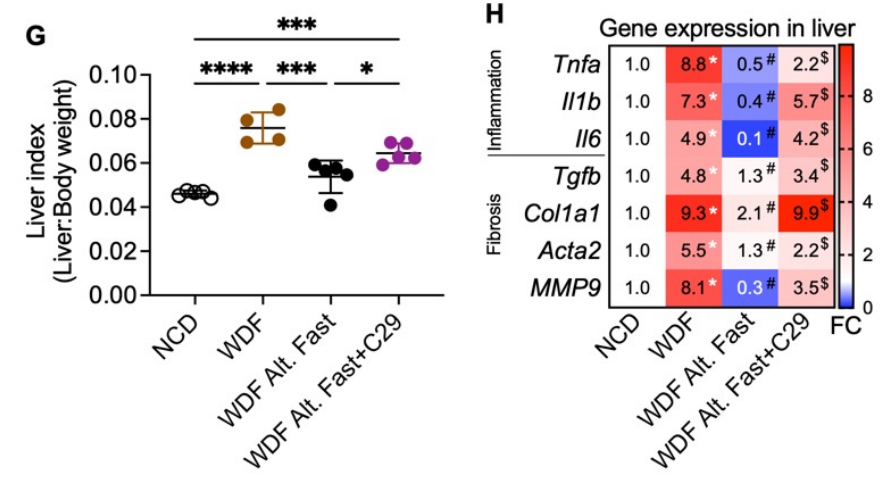

B Mice liver tissue
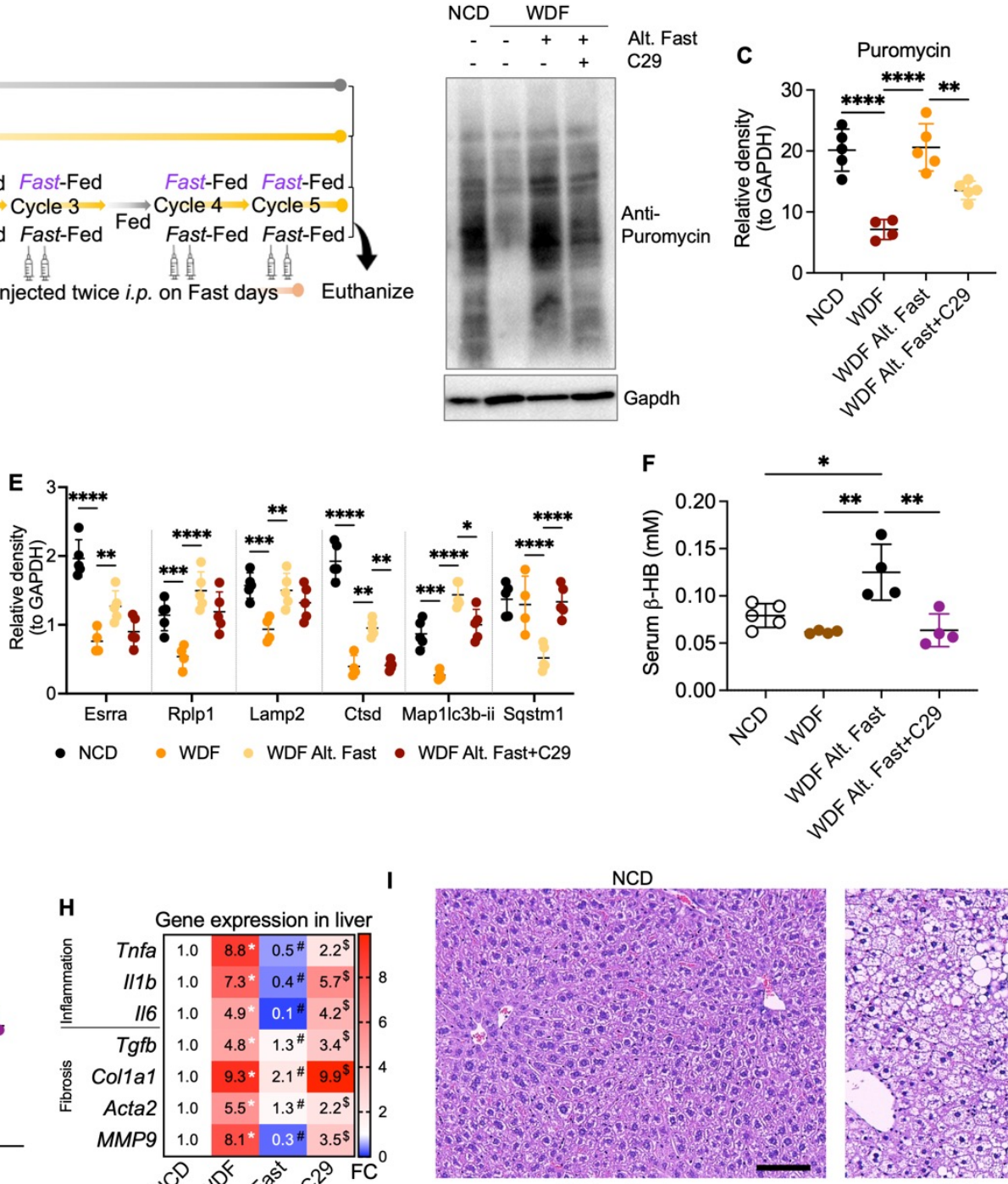

$\mathrm{WDF}+$ Alt. Fast
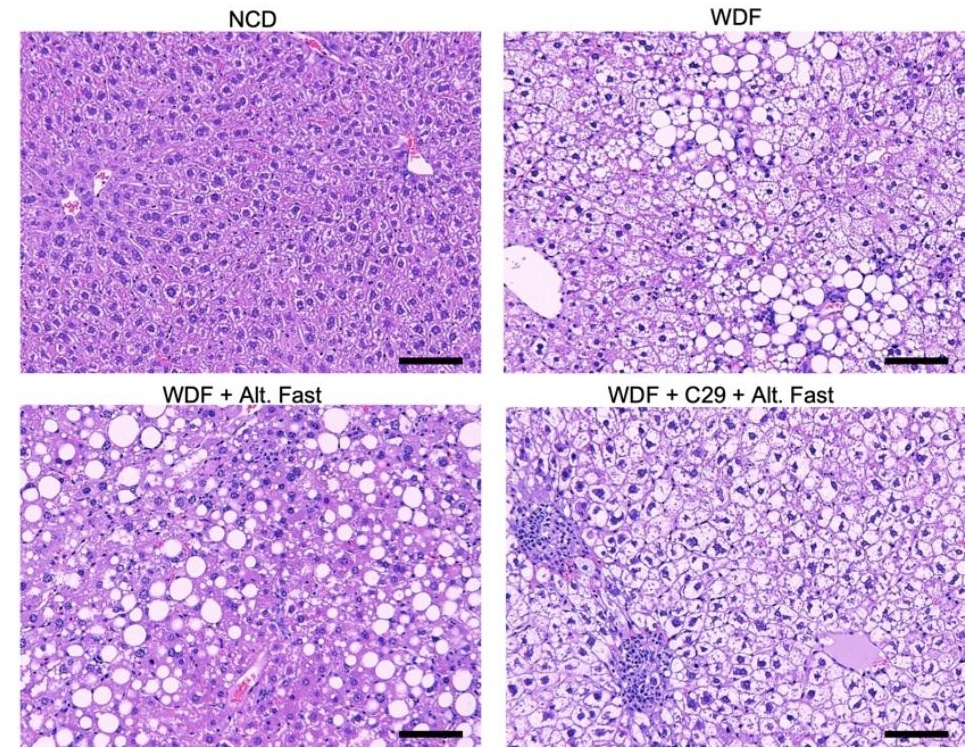

WDF + C29 + Alt. Fast

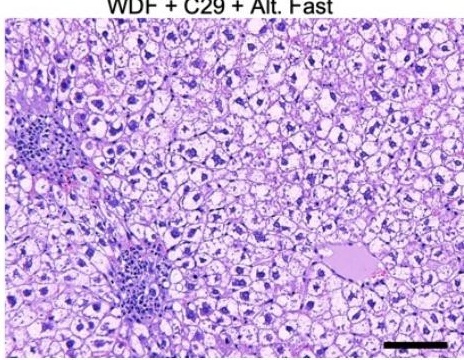

